\title{
Slavnov-Taylor identities in Coulomb gauge Yang-Mills theory
}

\author{
P. Watson and H. Reinhardt \\ Institut für Theoretische Physik, Universität Tübingen, \\ Auf der Morgenstelle 14, D-72076 Tübingen, Deutschland
}

\begin{abstract}
The Slavnov-Taylor identities of Coulomb gauge Yang-Mills theory are derived from the (standard, second order) functional formalism. It is shown how these identities form closed sets from which one can in principle fully determine the Green's functions involving the temporal component of the gauge field without approximation, given appropriate input.

PACS numbers: 11.15.Tk,12.38.Aw
\end{abstract}

\section{INTRODUCTION}

The issue of color confinement in quantum chromodynamics [QCD], widely accepted as the theory of the strong interaction, is a longstanding problem both conceptually and quantitatively. One necessary prerequisite for color confinement is the conservation of color charge: a 'leaky' system clearly cannot be confining. Within the framework of functional field-theoretic methods in nonabelian theories (i.e., QCD), expressed in terms of local Green's functions, the Slavnov-Taylor identities $[1,2]$ are the consequence of this charge conservation.

The Slavnov-Taylor identities come in various guises and have been applied to many problems, in particular to the nonperturbative study of the Dyson-Schwinger equations. For example, in quantum electrodynamics [QED] where the Slavnov-Taylor identities reduce to the Ward identity, their study led to the Ball-Chiu vertex [3] with its subsequent improvement, the Curtis-Pennington vertex [4], which have been used to study dynamical mass generation (see for example Refs. $[5,6])$. In axial gauge Yang-Mills theory, the Slavnov-Taylor identities [7] have been used to study the gluon propagator Dyson-Schwinger equation, with the inference that the propagator diverges as $1 / q^{4}$ in the infrared (one potential signal for confinement) [8]. This result is however, not without ambiguity [9]. In Landau gauge YangMills theory, the Mandelstam approximation [10] (see also Ref. [11]), where ghost contributions to the three-gluon vertex Slavnov-Taylor identity are neglected, one can also obtain an infrared enhanced gluon propagator. The above examples can be colloquially referred to as Schwinger-Dyson studies (these are early studies and a quirk of history has subsequently resulted in the reordering of the names) and they share two common features: the utilization of the Slavnov-Taylor identities and either require no, or assume no ghost contributions.

In attempting to further the Mandelstam approximation to Landau gauge Yang-Mills theory by including the ghost contributions, a startling new phenomenon emerged — infrared ghost dominance [12] (see also [6, 13] for contemporary reviews). The starting point was an approximation to the Slavnov-Taylor identity for the ghost-gluon vertex. This approximation was shown to be inconsistent with perturbation theory [14], but this turned out to be unimportant: using general arguments one can show that the tree-level part of the ghost-gluon vertex is the important ingredient [15]. Indeed, there are indications that the dressing of the ghost-gluon vertex function is only slight [16]. The results of this infrared ghost dominance can be summarized as follows: the gluon propagator is infrared suppressed, whereas the ghost propagator is infrared enhanced. In the context of confinement, the results imply positivity violation (formalized in the Oehme-Zimmermann superconvergence relations [17]) and are in agreement with the Kugo-Ojima [18] and Gribov-Zwanziger [19-21] confinement scenarios. These results have been contested (see for example Ref. [22]) by appealing to the Slavnov-Taylor identity for the three-gluon vertex, applying the further assumption that certain dressing functions that compose the ghost-gluon vertex kernel remain infrared finite and with the conclusion that the ghost propagator is not infrared enhanced. The existence of such additional solutions has been verified in Ref. [23] (this type of solution was incidentally also observed in Ref. [24] within the canonical approach to Coulomb gauge YangMills theory [25]): it was concluded however that the Slavnov-Taylor identities alone cannot discriminate between the two types of solution and that one needs explicit additional input (the authors arguing that the infrared enhanced ghost solution is preferred).

From the above (admittedly historically incomplete discussion of) works, several lessons emerge. Firstly, the Slavnov-Taylor identities play a central role in nonperturbative Dyson-Schwinger studies since they provide information about the higher $n$-point functions that enter the Dyson-Schwinger equations. Second, in all the above studies, the Slavnov-Taylor identities relate the 4 -vector contraction of a vertex to some combination of inverse propagators (proper two-point functions). This contraction means that only part of the vertex is constrained by the identity and it is not necessarily that part which enters the Dyson-Schwinger equations. One either assumes that the 'transverse' vertex (i.e., that part not constrained by the Slavnov-Taylor identity) can be neglected or considers more sophisticated input to complete the closure of the system such that the Dyson-Schwinger equations can be solved (e.g., the Curtis-Pennington vertex uses multiplicative renormalizability constraints [4]). Third, even if the Slavnov-Taylor 
identities are applied to close the Dyson-Schwinger equations, the solution (and in particular the infrared behavior) may not be uniquely specified and requires additional input, as demonstrated in Ref. [23]. These latter two points are emphatically not intended as a criticism of the Dyson-Schwinger approach, merely as reminders of some of the problems encountered (confinement is after all, not entirely trivial) and in this paper, we will see what Coulomb gauge has to say about the subject.

The importance of Coulomb gauge in studying nonperturbative QCD was recognized early on, as was the inherent difficulty in technical calculation for such noncovariant gauges [26]. The significance of Coulomb gauge is based in the observation that in this gauge, the system reduces naturally to physical degrees of freedom (explicitly demonstrated in Ref. [21]). Given that the Slavnov-Taylor identities are the expression of charge conservation as applied to Green's functions, there exists a clear motivation to derive them in Coulomb gauge. In addition, there has recently been much technical progress in the Coulomb gauge functional formalism that provides the background to the study of the Dyson-Schwinger equations: the derivation of the Dyson-Schwinger equations themselves [27, 28], their oneloop perturbative analysis [28-30], the resolution of more formal aspects of the (incomplete) gauge-fixing and the existence of a conserved, vanishing total charge (and the absence of the infamous Coulomb gauge energy divergences) $[31,32]$. With the Slavnov-Taylor identities, most of the components required for at least an initial study of the Dyson-Schwinger equations are in place.

In this paper, we thus derive the Slavnov-Taylor identities of Coulomb gauge Yang-Mils theory in the second order functional formalism and discuss some of their immediate consequences. In Section 2, the basic formulation of the functional formalism and the Gauss-BRST invariance is presented. The Slavnov-Taylor identities for the two-point functions are derived in detail in Section 3. In Section 4, the identities for the vertex functions are derived and it is shown how these identities form closed sets such that the temporal Green's functions may be obtained as their solution. Section 5 is concerned with the possibility of extracting unambiguous information about Green's functions from the identities in the infrared. The paper concludes with a discussion of various aspects of the identities. For the convenience of the reader, selected relevant results from earlier works are listed in Appendix A. Lengthy configuration space expressions, important to the derivation of the Slavnov-Taylor identities but not to the narrative of the paper are relegated to Appendix B.

\section{FUNCTIONAL FORMALISM AND GAUSS-BRST INVARIANCE}

Let us begin by considering Yang-Mills theory in the functional formalism. We will use the notation and conventions established in $[27,28]$. We work in Minkowski space with metric $g_{\mu \nu}=\operatorname{diag}(1,-\overrightarrow{1})$. Roman subscripts $(i, j, \ldots)$ denote spatial indices and superscripts $(a, b, \ldots)$ denote color indices. We will often write configuration space coordinates $(x, y, \ldots)$ as subscripts where no confusion arises.

The Yang-Mills action is defined as

$$
\mathcal{S}_{Y M}=\int d^{4} x\left[-\frac{1}{4} F_{\mu \nu}^{a} F^{a \mu \nu}\right]
$$

where the (antisymmetric) field strength tensor $F$ is given in terms of the gauge field $A_{\mu}^{a}$ :

$$
F_{\mu \nu}^{a}=\partial_{\mu} A_{\nu}^{a}-\partial_{\nu} A_{\mu}^{a}+g f^{a b c} A_{\mu}^{b} A_{\nu}^{c}
$$

In the above, the $f^{a b c}$ are the structure constants of the $S U\left(N_{c}\right)$ group whose generators obey $\left[T^{a}, T^{b}\right]=\imath f^{a b c} T^{c}$. The Yang-Mills action is invariant under a local $S U\left(N_{c}\right)$ gauge transform characterized by the parameter $\theta_{x}^{a}$ :

$$
U_{x}=\exp \left\{-\imath \theta_{x}^{a} T^{a}\right\}
$$

such that for infinitesimal $\theta_{x}^{a}$, the gauge field transforms as

$$
A_{\mu}^{a} \rightarrow A_{\mu}^{\prime a}=A_{\mu}^{a}-\frac{1}{g} \hat{D}_{\mu}^{a b} \theta^{b}
$$

with the covariant derivative in the adjoint color representation given by

$$
\hat{D}_{\mu}^{a c}=\delta^{a c} \partial_{\mu}+g f^{a b c} A_{\mu}^{b} .
$$

In terms of the temporal and spatial components, the above transform reads (we rewrite the temporal component $A_{0}$ as $\sigma$ )

$$
\begin{aligned}
\sigma^{a} \rightarrow \sigma^{\prime a} & =\sigma^{a}-\frac{1}{g} \partial_{0} \theta^{a}-f^{a b c} \sigma^{b} \theta^{c} \\
\vec{A}^{a} \rightarrow \vec{A}^{\prime a} & =\vec{A}^{a}+\frac{1}{g} \vec{\nabla} \theta^{a}-f^{a b c} \vec{A}^{b} \theta^{c}
\end{aligned}
$$


Consider the functional integral

$$
Z=\int \mathcal{D} \Phi \exp \left\{\imath \mathcal{S}_{Y M}\right\}
$$

where $\mathcal{D} \Phi$ denotes the functional integration measure for the collection of all fields. Since the action is invariant under gauge transformations, $Z$ is divergent by virtue of the integration over the gauge group. To overcome this problem we use the Faddeev-Popov technique and introduce a gauge-fixing term along with an associated ghost term [33]. Using a Lagrange multiplier field, $\lambda^{a}$, to implement the gauge-fixing, in Coulomb gauge $\left(\vec{\nabla} \cdot \overrightarrow{A^{a}}=0\right)$ we can then write

$$
Z=\int \mathcal{D} \Phi \exp \left\{\imath \mathcal{S}_{Y M}+\imath \mathcal{S}_{F P}\right\}, \quad \mathcal{S}_{F P}=\int d^{4} x\left[-\lambda^{a} \vec{\nabla} \cdot \vec{A}^{a}-\bar{c}^{a} \vec{\nabla} \cdot \vec{D}^{a b} c^{b}\right]
$$

where $\bar{c}^{a}$ and $c^{b}$ are the Grassmann-valued ghost fields. The new term in the action, $\mathcal{S}_{F P}$, is invariant under the GaussBecchi-Rouet-Stora-Tyutin [Gauss-BRST] transform [21] whereby the infinitesimal, spacetime dependent gauge parameter $\theta_{x}^{a}$ is factorized into two Grassmann-valued components: $\theta_{x}^{a}=c_{x}^{a} \delta \lambda_{t}$, where $\delta \lambda_{t}$ is the time-dependent infinitesimal variation (not to be confused with the colored Lagrange multiplier field $\lambda^{a}$ ). The Gauss-BRST transform is peculiar to Coulomb gauge - the time-dependent variation is allowed simply because the gauge-fixing does not involve any explicit time-derivatives. The variations of the new fields read:

$$
\delta \bar{c}_{x}^{a}=\frac{1}{g} \lambda_{x}^{a} \delta \lambda_{t}, \quad \delta c_{x}^{a}=-\frac{1}{2} f^{a b c} c_{x}^{b} c_{x}^{c} \delta \lambda_{t}, \quad \delta \lambda_{x}^{a}=0 .
$$

By including a source term, the generating functional is given by

$$
Z[J]=\int \mathcal{D} \Phi \exp \left\{\imath \mathcal{S}_{Y M}+\imath \mathcal{S}_{F P}+\imath \mathcal{S}_{s}\right\}
$$

where

$$
\mathcal{S}_{s}=\int d^{4} x\left[\rho^{a} \sigma^{a}+\vec{J}^{a} \cdot \vec{A}^{a}+\bar{c}^{a} \eta^{a}+\bar{\eta}^{a} c^{a}+\xi^{a} \lambda^{a}\right] .
$$

Regarding the Gauss-BRST transform as a change of integration variables under which the generating functional is invariant, noting that the associated Jacobian factor is trivial [27] and only the source term varies, we deduce that

$$
\begin{aligned}
0= & \left.\int \mathcal{D} \Phi \frac{\delta}{\delta\left[\imath \delta \lambda_{t}\right]} \exp \left\{\imath \mathcal{S}_{Y M}+\imath \mathcal{S}_{F P}+\imath \mathcal{S}_{s}+\imath \delta \mathcal{S}_{s}\right\}\right|_{\delta \lambda_{t}=0} \\
= & \int \mathcal{D} \Phi \exp \left\{\imath \mathcal{S}_{Y M}+\imath \mathcal{S}_{F P}+\imath \mathcal{S}_{s}\right\} \int d^{4} x \delta\left(t-x_{0}\right) \times \\
& {\left[-\frac{1}{g}\left(\partial_{x}^{0} \rho_{x}^{a}\right) c_{x}^{a}+f^{a b c} \rho_{x}^{a} \sigma_{x}^{b} c_{x}^{c}-\frac{1}{g} J_{i x}^{a} \nabla_{i x} c_{x}^{a}+f^{a b c} J_{i x}^{a} A_{i x}^{b} c_{x}^{c}+\frac{1}{g} \lambda_{x}^{a} \eta_{x}^{a}+\frac{1}{2} f^{a b c} \bar{\eta}_{x}^{a} c_{x}^{b} c_{x}^{c}\right] . }
\end{aligned}
$$

This equation is the starting point for deriving the Slavnov-Taylor identities. Notice the $\delta\left(t-x_{0}\right)$ constraint, which arises because of the time-dependent variation $\delta \lambda_{t}$ and is characteristic to the Gauss-BRST transform. It leads eventually to a nontrivial energy injection into the Slavnov-Taylor identities which is not present in the covariant gauge case.

It is pertinent at this stage to discuss some nontrivial points associated with the above. Coulomb gauge is in fact not a complete gauge. Even after adding the gauge-fixing terms, the functional integral, Eq. (2.8), still contains zero-modes generated by purely temporal gauge transforms (and for that matter, global transforms too). Explicitly separating these temporal zero-modes within the Faddeev-Popov procedure, one can formally show (within the first order formalism, but since this is connected to the second order formalism used here via identities, the same conclusions apply) that the total color charge of the system is constrained to be conserved and vanishing [32]. The above expression, Eq. (2.12), is the dynamical statement of color charge conservation - it shows how the external sources must be arranged in accordance with the gauge symmetry of the underlying theory. In the two differing contexts, the incompleteness of the gauge plays a central role: the formal isolation of the zero-modes gives the total charge constraint and the time-dependent variation $\left(\delta \lambda_{t}\right)$ will give (later) the extra temporal scale. We will discuss this connection at the end.

Note also that the presence of the temporal zero-modes precludes deriving a similar expression to Eq. (2.12) above by considering a purely time dependent (spatially independent) full gauge transform, i.e., $\theta(t, \vec{x}) \rightarrow \theta(t)$, since the 
functional integration is ill-defined. In such a case, the identity gives a resultant $\Gamma_{\sigma \sigma}$ Green's function (see later for details of the definition) with a dressing function that vanishes at zero momentum and this would hold even at tree-level which clearly contradicts the perturbative behavior for which the dressing function is unity (see Ref. [28] or Appendix A). The reason for this is that when considering the gauge-variant Green's functions, the integration over the gauge group associated with the zero-modes averages such quantities to zero. For the Gauss-BRST transform considered here, the spatial dependence of the ghost-field ensures that this problem is not encountered (since the gauge-fixing part of the action can be rewritten in terms of spatial derivatives of the ghost-field, they can always be implicitly defined as having no spatially constant component).

So far, the generating functional, $Z[J]$, generates all Green's functions, connected and disconnected. The generating functional of connected Green's functions is $W[J]$ where

$$
Z[J]=e^{W[J]} .
$$

We define the classical fields to be

$$
\Phi_{\alpha}=\frac{1}{Z} \int \mathcal{D} \Phi \Phi_{\alpha} \exp \imath \mathcal{S}=\frac{1}{Z} \frac{\delta Z}{\delta \imath J_{\alpha}}
$$

(we use the same notation for both the classical and quantum fields since no confusion will arise). The generating functional of proper Green's functions is the effective action, $\Gamma$, which is a functional of the classical fields and is defined through a Legendre transform of $W$ :

$$
\Gamma[\Phi]=W[J]-\imath J_{\alpha} \Phi_{\alpha} .
$$

In the above, we use a compact notation for the sources and fields: a generic field is denoted $\Phi_{\alpha}$, with source $J_{\alpha}$ and with the index $\alpha$ standing for all attributes of the field in question (including its type); further, summation over all discrete indices and integration over all continuous arguments is implicitly understood. We introduce a bracket notation for derivatives of $W$ with respect to sources and of $\Gamma$ with respect to classical fields (no confusion arises since the two sets of derivatives are never mixed):

$$
<\imath J_{\alpha}>=\frac{\delta W}{\delta \imath J_{\alpha}}, \quad<\imath \Phi_{\alpha}>=\frac{\delta \Gamma}{\delta \imath \Phi_{\alpha}} .
$$

We can now rewrite Eq. (2.12) as

$$
\begin{aligned}
0= & \int d^{4} x \delta\left(t-x_{0}\right)\left\{\frac{1}{g}\left(\partial_{x}^{0}<\imath \sigma_{x}^{a}>\right) c_{x}^{a}-f^{a b c}<\imath \sigma_{x}^{a}>\sigma_{x}^{b} c_{x}^{c}+\frac{1}{g}<\imath A_{i x}^{a}>\nabla_{i x} c_{x}^{a}-f^{a b c}<\imath A_{i x}^{a}>A_{i x}^{b} c_{x}^{c}-\frac{1}{g} \lambda_{x}^{a}<\imath \bar{c}_{x}^{a}>\right. \\
& \left.+\frac{1}{2} f^{a b c}<\imath c_{x}^{a}>c_{x}^{b} c_{x}^{c}-f^{a b c}<\imath \sigma_{x}^{a}><\imath \rho_{x}^{b} \bar{\eta}_{x}^{c}>-f^{a b c}<\imath A_{i x}^{a}><\imath J_{i x}^{b} \imath \bar{\eta}_{x}^{c}>+\frac{1}{2} f^{a b c}<\imath c_{x}^{a}><\imath \bar{\eta}_{x}^{b} \imath \bar{\eta}_{x}^{c}>\right\} .
\end{aligned}
$$

Knowing the functional form of the ghost equation of motion [28],

$$
<\imath \bar{c}_{x}^{a}>=-\nabla_{x}^{2} c_{x}^{a}+g f^{a b c} \nabla_{i x}\left[<\imath J_{i x}^{b} \imath \bar{\eta}_{x}^{c}>+A_{i x}^{b} c_{x}^{c}\right],
$$

allows us to write

$$
\begin{aligned}
& \int d^{4} x \delta\left(t-x_{0}\right) \frac{1}{g}<\imath A_{i x}^{a}>\nabla_{i x} c_{x}^{a}=\int d^{4} x \delta\left(t-x_{0}\right) \frac{1}{g}\left[\frac{\nabla_{i x}}{\left(-\nabla_{x}^{2}\right)}<\imath A_{i x}^{a}>\right] \nabla_{x}^{2} c_{x}^{a} \\
& =\int d^{4} x \delta\left(t-x_{0}\right)\left\{-\frac{1}{g}\left[\frac{\nabla_{i x}}{\left(-\nabla_{x}^{2}\right)}<\imath A_{i x}^{a}>\right]<\imath \bar{c}_{x}^{a}>-f^{a b c}<\imath A_{i x}^{a}>\frac{\nabla_{i x} \nabla_{j x}}{\left(-\nabla_{x}^{2}\right)}\left[<\imath J_{j x}^{b} \imath \bar{\eta}_{x}^{c}>+A_{j x}^{b} c_{x}^{c}\right]\right\} .
\end{aligned}
$$

Inserting the above into Eq. (2.17) then gives

$$
\begin{aligned}
0= & \int d^{4} x \delta\left(t-x_{0}\right)\left\{\frac{1}{g}\left(\partial_{x}^{0}<\imath \sigma_{x}^{a}>\right) c_{x}^{a}-f^{a b c}<\imath \sigma_{x}^{a}>\sigma_{x}^{b} c_{x}^{c}-\frac{1}{g}\left[\frac{\nabla_{i x}}{\left(-\nabla_{x}^{2}\right)}<\imath A_{i x}^{a}>\right]<\imath \bar{c}_{x}^{a}>\right. \\
& -f^{a b c}<\imath A_{i x}^{a}>t_{i j}(\vec{x}) A_{j x}^{b} c_{x}^{c}-\frac{1}{g} \lambda_{x}^{a}<\imath \bar{c}_{x}^{a}>+\frac{1}{2} f^{a b c}<\imath c_{x}^{a}>c_{x}^{b} c_{x}^{c}-f^{a b c}<\imath \sigma_{x}^{a}><\imath \rho_{x}^{b} \imath \bar{\eta}_{x}^{c}> \\
& \left.-f^{a b c}<\imath A_{i x}^{a}>t_{i j}(\vec{x})<\imath J_{j x}^{b} \imath \bar{\eta}_{x}^{c}>+\frac{1}{2} f^{a b c}<\imath c_{x}^{a}><\imath \bar{\eta}_{x}^{b} \imath \bar{\eta}_{x}^{c}>\right\}
\end{aligned}
$$


where $t_{i j}(\vec{x})=\delta_{i j}+\nabla_{i x} \nabla_{j x} /\left(-\nabla_{x}^{2}\right)$ is the transverse projector in configuration space.

Before considering the full content of the Slavnov-Taylor identities arising from Eq. (2.20), let us show that functional derivatives with respect to the Lagrange multiplier field, $\lambda_{x}^{a}$, play no further role. The field equation of motion for $\lambda_{x}^{a}$, valid in the presence of sources reads [28]

$$
<\imath \lambda_{x}^{a}>=-\nabla_{i x} A_{i x}^{a}
$$

such that the only non-zero functional derivative of $\left\langle\imath \lambda_{x}^{a}\right\rangle$ is

$$
<\imath A_{j y}^{b} \imath \lambda_{x}^{a}>=\imath \delta^{b a} \nabla_{j x} \delta(y-x) .
$$

All other functional derivatives of $\left\langle\imath \lambda_{x}^{a}\right\rangle$ vanish, even in the presence of sources. Taking the functional derivative of Eq. (2.20) with respect to $\imath \lambda_{z}^{d}$ gives

$$
\begin{aligned}
0= & \int d^{4} x \delta\left(t-x_{0}\right)\left\{\frac{\imath}{g} \delta(z-x)<\imath \bar{c}_{x}^{d}>-\frac{1}{g}\left[\frac{\nabla_{i x}}{\left(-\nabla_{x}^{2}\right)}<\imath \lambda_{z}^{d} \imath A_{i x}^{a}>\right]<\imath \bar{c}_{x}^{a}>\right. \\
& -f^{a b c}<\imath \lambda_{z}^{d} \imath A_{i x}^{a}>t_{i j}(\vec{x}) A_{j x}^{b} c_{x}^{c}-f^{a b c}<\imath \lambda_{z}^{d} \imath A_{i x}^{a}>t_{i j}(\vec{x})<\imath J_{j x}^{b} \imath \bar{\eta}_{x}^{c}> \\
& \left.-f^{a b c}<\imath \sigma_{x}^{a}>\frac{\delta}{\delta \imath \lambda_{z}^{d}}<\imath \rho_{x}^{b} \imath \bar{\eta}_{x}^{c}>-f^{a b c}<\imath A_{i x}^{a}>t_{i j}(\vec{x}) \frac{\delta}{\delta \imath \lambda_{z}^{d}}<\imath J_{j x}^{b} \imath \bar{\eta}_{x}^{c}>+\frac{1}{2} f^{a b c}<\imath c_{x}^{a}>\frac{\delta}{\delta \imath \lambda_{z}^{d}}<\imath \bar{\eta}_{x}^{b} \imath \bar{\eta}_{x}^{c}>\right\} .
\end{aligned}
$$

Now, taking partial functional derivatives of the Legendre transform, Eq. (2.15), one can show that

$$
\begin{aligned}
\frac{\delta}{\delta \imath \lambda_{z}^{d}}<\imath J_{\alpha} \imath J_{\beta}> & \sim<\imath J_{\alpha} \imath J_{\kappa}><\imath \Phi_{\kappa} \imath \lambda_{z}^{d} \imath \Phi_{\gamma}><\imath J_{\gamma} \imath J_{\beta}> \\
& =0
\end{aligned}
$$

since all three-point proper functions involving functional derivatives with respect to $\imath \lambda_{z}^{d}$ vanish. Further using the result Eq. (2.22) we arrive at

$$
0=\int d^{4} x \delta\left(t-x_{0}\right)\left\{\frac{\imath}{g}\left[\delta(z-x)-\frac{\nabla_{i x} \nabla_{i z}}{\left(-\nabla_{x}^{2}\right)} \delta(x-z)\right]<\imath \bar{c}_{x}^{d}>-\imath f^{d b c}\left[\nabla_{i z} \delta(x-z)\right] t_{i j}(\vec{x})\left[A_{j x}^{b} c_{x}^{c}+<\imath J_{j x}^{b} \imath \bar{\eta}_{x}^{c}>\right]\right\} .
$$

Knowing that $\nabla_{i z} \delta(x-z)=-\nabla_{i x} \delta(x-z)$ and using integration by parts on the last term, the above expression is a trivial identity. Thus, even in the presence of sources, functional derivatives of Eq. (2.20) with respect to $\imath \lambda_{z}^{d}$ give rise to an identity from which no further information can be obtained and we can set the classical field $\lambda_{x}^{a}=0$ (except for within partial derivatives used in conjunction with the Legendre transform) and ignore the Lagrange multiplier field from now on.

Equation (2.20) is Grassmann valued and to proceed, we must first take the functional derivative with respect to $\imath c_{z}^{d}$. Since we will be taking further derivatives, fields/sources must be retained (with the exception of $\lambda$ as discussed previously). The subsequent Slavnov-Taylor identities are thus functional derivatives of

$$
\begin{aligned}
0= & \int d^{4} x \delta\left(t-x_{0}\right)\left\{-\frac{\imath}{g}\left[\partial_{x}^{0}<\imath c_{z}^{d} \imath \sigma_{x}^{a}>\right] \imath c_{x}^{a}-\frac{\imath}{g}\left[\partial_{x}^{0}<\imath \sigma_{x}^{d}>\right] \delta(z-x)\right. \\
& -\frac{1}{g}\left[\frac{\nabla_{i x}}{\left(-\nabla_{x}^{2}\right)}<\imath c_{z}^{d} \imath A_{i x}^{a}>\right]<\imath \bar{c}_{x}^{a}>+\frac{1}{g}\left[\frac{\nabla_{i x}}{\left(-\nabla_{x}^{2}\right)}<\imath A_{i x}^{a}>\right]<\imath \bar{c}_{x}^{a} \imath c_{z}^{d}> \\
& -f^{a b c}<\imath c_{z}^{d} \imath \sigma_{x}^{a}>\left[<\imath \rho_{x}^{b} \imath \bar{\eta}_{x}^{c}>-\imath \sigma_{x}^{b} \imath c_{x}^{c}\right]-f^{a b c}<\imath \sigma_{x}^{a}>\left[\frac{\delta}{\delta \imath c_{z}^{d}}<\imath \rho_{x}^{b} \bar{\eta}_{x}^{c}>-\imath \sigma_{x}^{b} \delta^{d c} \delta(z-x)\right] \\
& -f^{a b c}<\imath c_{z}^{d} \imath A_{i x}^{a}>t_{i j}(\vec{x})\left[<\imath J_{j x}^{b} \imath \bar{\eta}_{x}^{c}>-\imath A_{j x}^{b} \imath c_{x}^{c}\right]-f^{a b c}<\imath A_{i x}^{a}>t_{i j}(\vec{x})\left[\frac{\delta}{\delta \imath c_{z}^{d}}<\imath J_{j x}^{b} \imath \bar{\eta}_{x}^{c}>-\imath A_{j x}^{b} \delta^{d c} \delta(z-x)\right] \\
& \left.+\frac{1}{2} f^{a b c}<\imath c_{z}^{d} \imath c_{x}^{a}>\left[<\imath \bar{\eta}_{x}^{b} \bar{\eta}_{x}^{c}>-\imath c_{x}^{b} \imath c_{x}^{c}\right]-\frac{1}{2} f^{a b c}<\imath c_{x}^{a}>\left[\frac{\delta}{\delta \imath c_{z}^{d}}<\imath \bar{\eta}_{x}^{b} \imath \bar{\eta}_{x}^{c}>-2 \delta^{d b} \delta(z-x) \imath c_{x}^{c}\right]\right\} .
\end{aligned}
$$

\section{SLAVNOV-TAYLOR IDENTITIES: TWO-POINT FUNCTIONS}

The first of the Slavnov-Taylor identities is generated by taking the functional derivative of Eq. (2.26) with respect to $\imath \sigma_{w}^{e}$ and setting sources to zero. The resulting equation reads

$$
0=\int d^{4} x \delta\left(t-x_{0}\right)\left\{-\frac{\imath}{g}\left[\partial_{x}^{0}<\imath \sigma_{w}^{e} \imath \sigma_{x}^{d}>\right] \delta(z-x)+\frac{1}{g}\left[\frac{\nabla_{i x}}{\left(-\nabla_{x}^{2}\right)}<\imath \sigma_{w}^{e} \imath A_{i x}^{a}>\right]<\imath \bar{c}_{x}^{a} \imath c_{z}^{d}>\right.
$$




$$
\left.-f^{a b c}<\imath \sigma_{w}^{e} \imath \sigma_{x}^{a}>\frac{\delta}{\delta \imath c_{z}^{d}}<\imath \rho_{x}^{b} \imath \bar{\eta}_{x}^{c}>\left.\right|_{J=0}-f^{a b c}<\imath \sigma_{w}^{e} \imath A_{i x}^{a}>t_{i j}(\vec{x}) \frac{\delta}{\delta \imath c_{z}^{d}}<\imath J_{j x}^{b} \imath \bar{\eta}_{x}^{c}>\left.\right|_{J=0}\right\}
$$

This equation is best expressed in momentum space. We define the momentum space two-point Green's functions via their respective Fourier transforms

$$
<\imath \sigma_{w}^{a} \imath \sigma_{x}^{b}>=\int d k e^{-\imath k \cdot(w-x)} \Gamma_{\sigma \sigma}^{a b}\left(k_{0}, \vec{k}\right)
$$

(similarly for $\Gamma_{A A}$ etc. and also for the propagators $W_{A A}$ etc.) where the arguments of the momentum space functions reflect their noncovariant nature, we impose translational invariance in the usual way and $d k=d^{4} k /(2 \pi)^{4}$. We also define

$$
\begin{aligned}
f^{a b c} \frac{\delta}{\delta \imath c_{z}^{d}}<\imath \rho_{x}^{b} \imath \bar{\eta}_{x}^{c}>\left.\right|_{J=0} & =\int d k e^{-\imath k \cdot(x-z)} \tilde{\Sigma}_{\sigma ; \bar{c} c}^{a d}\left(k_{0}, \vec{k}\right), \\
f^{a b c} \frac{\delta}{\delta \imath c_{z}^{d}}<\imath J_{j x}^{b} \imath \bar{\eta}_{x}^{c}>\left.\right|_{J=0} & =\int d k e^{-\imath k \cdot(x-z)} \tilde{\Sigma}_{A ; \bar{c} c j}^{a d}\left(k_{0}, \vec{k}\right) .
\end{aligned}
$$

The latter two definitions and notations may at first sight appear somewhat artificial. In fact, as will be shortly justified, their exact form in terms of standard Green's functions is irrelevant since neither term will contribute. However, in the remainder of the paper, similar (but increasingly complicated) objects will occur so some explanation is in order. The Dyson-Schwinger equations are derived as functional derivatives of the field equations of motion and this gives rise to the familiar loop expressions. For example (taken from Ref. [28]),

$$
\frac{\delta}{\delta \imath A_{j w}^{f}} \int d^{4} y d^{4} z \Gamma_{\bar{c} c A i}^{(0) b c a}(y, z, x)<i \bar{\eta}_{z}^{c} \imath \eta_{y}^{b}>\longrightarrow \int d \omega \Gamma_{\bar{c} c A i}^{(0) b c a}(\omega-k,-\omega, k) W_{\bar{c} c}^{c d}(\omega) \Gamma_{\bar{c} c A j}^{d e f}(\omega, k-\omega,-k) W_{\bar{c} c}^{e b}(\omega-k)
$$

gives the ghost loop term of the Dyson-Schwinger equation for $\Gamma_{A A}\left(W_{\bar{c} c}\right.$ is the ghost propagator, $\Gamma_{\bar{c} c A}$ is the spatial ghost-gluon vertex and $\Gamma_{\bar{c} c A}^{(0)}$ is the tree-level counterpart). Importantly, all occurrences of objects such as $\left\langle\imath \bar{\eta}_{z}^{c} \imath \eta_{y}^{b}>\right.$ in the field equations of motion have an associated tree-level vertex - these terms originate directly from the interaction terms of the original action such that the functional derivatives have a clear meaning as loops. However, in the case of the (nonabelian) Slavnov-Taylor identities one has a different structure which arises from the Gauss-BRST transform (or indeed generally from the BRS transform) and is exemplified in Eq. (2.26). In this case, one has objects such as $\left\langle\imath \rho_{x}^{b} \lambda \bar{\eta}_{x}^{c}\right\rangle$ which must be functionally differentiated, but which are separated from any interaction type of factor. However, even without the interaction term, these functional derivatives do still have a partial meaning as loop expressions; it is simply that the tree-level vertex is missing (although the color factor and momentum conservation are present). We will denote such irregular expressions with a tilde (as in $\tilde{\Sigma}$ above or $\tilde{\Gamma}$ later) and since these terms are important to the arguments presented in this study, we will write out their explicit forms where necessary. As alluded to above, this type of pseudo-loop expression is common to the Slavnov-Taylor identities of nonabelian theories and in linear covariant gauges, there is a familiar example, referred to as the ghost-gluon scattering-like kernel which appears in the identity for the three-gluon vertex [34].

Returning to Eq. (3.1), we can now write

$$
\begin{aligned}
0=\int d q_{0} d k e^{-\imath q_{0}\left(t-z_{0}\right)-\imath k \cdot(w-z)} & \left\{\frac{k_{0}}{g} \Gamma_{\sigma \sigma}^{e d}\left(k_{0}, \vec{k}\right)-\frac{\imath}{g} \frac{k_{i}}{\vec{k}^{2}} \Gamma_{\sigma A i}^{e a}\left(k_{0}, \vec{k}\right) \Gamma_{\bar{c} c}^{a d}\left(q_{0}+k_{0}, \vec{k}\right)\right. \\
& \left.-\Gamma_{\sigma \sigma}^{e a}\left(k_{0}, \vec{k}\right) \tilde{\Sigma}_{\sigma ; \bar{c} c}^{a d}\left(q_{0}+k_{0}, \vec{k}\right)-\Gamma_{\sigma A i}^{e a}\left(k_{0}, \vec{k}\right) t_{i j}(\vec{k}) \tilde{\Sigma}_{A ; \bar{c} c j}^{a d}\left(k_{0}+q_{0}, \vec{k}\right)\right\} .
\end{aligned}
$$

In the above, notice how the $\delta\left(t-x_{0}\right)$ factor characterizing the Gauss-BRST transform leads to the energy insertion $q_{0}$ which breaks the energy flow through the various Green's functions and in particular how this insertion affects only the ghost functions. Since $t, w$ and $z$ are arbitrary, we can write down the momentum space Slavnov-Taylor identity:

$$
\frac{k_{0}}{g} \Gamma_{\sigma \sigma}^{e d}\left(k_{0}, \vec{k}\right)=\frac{\imath}{g} \frac{k_{i}}{\vec{k}^{2}} \Gamma_{\sigma A i}^{e a}\left(k_{0}, \vec{k}\right) \Gamma_{\bar{c} c}^{a d}\left(q_{0}+k_{0}, \vec{k}\right)+\Gamma_{\sigma \sigma}^{e a}\left(k_{0}, \vec{k}\right) \tilde{\Sigma}_{\sigma ; \bar{c} c}^{a d}\left(q_{0}+k_{0}, \vec{k}\right)+\Gamma_{\sigma A i}^{e a}\left(k_{0}, \vec{k}\right) t_{i j}(\vec{k}) \tilde{\Sigma}_{A ; \bar{c} c j}^{a d}\left(k_{0}+q_{0}, \vec{k}\right) .
$$

Now consider the definition of $\tilde{\Sigma}_{A j ; \bar{c} c}$, given by Eq. (3.4): under a parity transform, the vector source $J_{j x}^{b}$ changes sign $[27]$ and we see that

$$
\tilde{\Sigma}_{A ; \bar{c} c j}^{a d}\left(k_{0},-\vec{k}\right)=-\tilde{\Sigma}_{A ; \bar{c} c j}^{a d}\left(k_{0}, \vec{k}\right)
$$


from which we can infer that

$$
t_{i j}(\vec{k}) \tilde{\Sigma}_{A ; \bar{c} c j}^{a d}\left(k_{0}+q_{0}, \vec{k}\right) \sim t_{i j}(\vec{k}) k_{j}=0
$$

such that the last term of Eq. (3.7) vanishes. Because the energy scale, $q_{0}$, is arbitrary, we can make a further translation $q_{0} \rightarrow-q_{0}-2 k_{0}$ and Eq. (3.7) becomes

$$
\frac{k_{0}}{g} \Gamma_{\sigma \sigma}^{e d}\left(k_{0}, \vec{k}\right)=\frac{\imath}{g} \frac{k_{i}}{\overrightarrow{k^{2}}} \Gamma_{\sigma A i}^{e a}\left(k_{0}, \vec{k}\right) \Gamma_{\bar{c} c}^{a d}\left(-q_{0}-k_{0}, \vec{k}\right)+\Gamma_{\sigma \sigma}^{e a}\left(k_{0}, \vec{k}\right) \tilde{\Sigma}_{\sigma ; \bar{c} c}^{a d}\left(-q_{0}-k_{0}, \vec{k}\right) .
$$

Knowing that the $\sigma$-field and $\rho$-source change sign under time-reversal, whereas the ghost-antighost pair is invariant [27], we have that

$$
\begin{aligned}
\tilde{\Sigma}_{\sigma ; \bar{c} c}^{a d}\left(-q_{0}-k_{0}, \vec{k}\right) & =-\tilde{\Sigma}_{\sigma ; \bar{c} c}^{a d}\left(q_{0}+k_{0}, \vec{k}\right), \\
\Gamma_{\bar{c} c}^{a d}\left(-q_{0}-k_{0}, \vec{k}\right) & =\Gamma_{\bar{c} c}^{a d}\left(q_{0}+k_{0}, \vec{k}\right)
\end{aligned}
$$

such that

$$
\frac{k_{0}}{g} \Gamma_{\sigma \sigma}^{e d}\left(k_{0}, \vec{k}\right)=\frac{\imath}{g} \frac{k_{i}}{\vec{k}^{2}} \Gamma_{\sigma A i}^{e a}\left(k_{0}, \vec{k}\right) \Gamma_{\bar{c} c}^{a d}\left(q_{0}+k_{0}, \vec{k}\right)-\Gamma_{\sigma \sigma}^{e a}\left(k_{0}, \vec{k}\right) \tilde{\Sigma}_{\sigma ; \bar{c} c}^{a d}\left(q_{0}+k_{0}, \vec{k}\right) .
$$

Comparing this with the original Eq. (3.7) above, we find that

$$
\tilde{\Sigma}_{\sigma ; \bar{c} c}^{a d}\left(q_{0}+k_{0}, \vec{k}\right)=0 .
$$

The Slavnov-Taylor identity now reads

$$
\frac{k_{0}}{g} \Gamma_{\sigma \sigma}^{e d}\left(k_{0}, \vec{k}\right)=\frac{\imath}{g} \frac{k_{i}}{\overrightarrow{k^{2}}} \Gamma_{\sigma A i}^{e a}\left(k_{0}, \vec{k}\right) \Gamma_{\bar{c} c}^{a d}\left(q_{0}+k_{0}, \vec{k}\right) .
$$

However, since $q_{0}$ is arbitrary, we can further state that the two-point ghost proper function $\Gamma_{\bar{c} c}$ is independent of energy, i.e.,

$$
\Gamma_{\bar{c} c}^{a d}\left(q_{0}+k_{0}, \vec{k}\right) \rightarrow \Gamma_{\bar{c} c}^{a d}(\vec{k})
$$

This was known to all orders in perturbation theory [27, 28], but it is reassuring that the result is confirmed nonperturbatively. We now have the final form of the first of the Slavnov-Taylor identities:

$$
\frac{k_{0}}{g} \Gamma_{\sigma \sigma}^{e d}\left(k_{0}, \vec{k}\right)=\frac{\imath}{g} \frac{k_{i}}{\vec{k}^{2}} \Gamma_{\sigma A i}^{e a}\left(k_{0}, \vec{k}\right) \Gamma_{\bar{c} c}^{a d}(\vec{k}) .
$$

Repeating the above analysis but starting by functionally differentiating Eq. (2.26) with respect to $\imath A_{k w}^{e}$ and setting sources to zero leads to the similar Slavnov-Taylor identity

$$
\frac{k_{0}}{g} \Gamma_{A \sigma k}^{e d}\left(k_{0}, \vec{k}\right)=\frac{\imath}{g} \frac{k_{i}}{\vec{k}^{2}} \Gamma_{A A k i}^{e a}\left(k_{0}, \vec{k}\right) \Gamma_{\bar{c} c}^{a d}(\vec{k}) .
$$

Now, since the energy, $k_{0}$, is a scalar quantity we see immediately from Eqs. (3.16) and (3.17) that the two-point proper Green's functions involving functional derivatives with respect to the temporal gluon field, $\sigma$, (these will be referred to as the temporal Green's functions) can be unambiguously expressed in terms of the ghost and (longitudinal) spatial gluon two-point proper Green's functions. (We will discuss the identities, Eqs. (3.16) and (3.17), and their general kinematical decompositions further in Section 5.) In a sense, up to the level of the two-point functions the field $\sigma$ has been eliminated from the system or equivalently has been integrated out of the functional form of the action. The two identities, Eqs. (3.16) and (3.17), have been derived previously from the standard BRS invariance, using perturbative arguments to eliminate the $\tilde{\Sigma}$ terms and were verified to one-loop order in perturbation theory [28]. The above derivation is however slightly superior since it makes reference only to symmetry arguments and does not rely on the implicit assumption that the all-orders resummation of perturbation theory is equivalent to the nonperturbative theory. Together, the two identities are the Coulomb gauge equivalent of the standard covariant gauge result that the longitudinal part of the gluon polarization is not dressed [1, 2]. This also highlights an important difference between Landau gauge and Coulomb gauge: in the former, the gluon polarization is transverse to the fourmomentum; in the latter, the gluon polarization ( $\Gamma_{A A}$ in our notation) is explicitly not transverse - instead, the spatially longitudinal, ghost and temporal two-point proper functions are all related. This connection is reminiscent of the quartet mechanism in the Kugo-Ojima confinement scenario [18]. This is hardly surprising since the Kugo-Ojima confinement scenario is based on the identification of a well-defined conserved color charge and the Slavnov-Taylor identities here are the dynamical expression of charge conservation. 


\section{SLAVNOV-TAYLOR IDENTITIES: VERTEX FUNCTIONS}

In this section, we study further functional derivatives of Eq. (2.26) and show that the resultant Slavnov-Taylor identities eventually form a closed set from which the temporal Green's functions (i.e., those with at least one external $\sigma$-leg) can be unambiguously derived. This closed set turns out to be somewhat extended and so, for clarity of presentation, we separate the various classes of functional derivatives according to how many pairs of ghost/antighost functional derivatives are present. By explicitly separating the Grassmann-valued fields, we may then modify our notation for the generic field $\Phi_{\alpha}$ or source $J_{\alpha}$ to be restricted to only the $\vec{A}$ or $\sigma$ fields/sources and their associated attributes which allows us to compactify the formalism.

\section{A. No further ghost derivatives}

Let us begin by functionally differentiating Eq. (2.26) twice with respect to $\imath \Phi_{\lambda(k) w}^{e}$ and $\imath \Phi_{\tau(l) v}^{f}$ where as mentioned above, the indices $\lambda(k)$ and $\tau(l)$ refer here to either the $A$-field with its associated spatial index ( $k$ or $l$ ) or to the $\sigma$-field with no associated spatial index. Setting sources to zero, the resulting equation reads:

$$
\begin{aligned}
0= & \int d^{4} x \delta\left(t-x_{0}\right)\left\{-\frac{\imath}{g}\left[\partial_{x}^{0}<\imath \Phi_{\tau(l) v}^{f} \imath \Phi_{\lambda(k) w}^{e} \imath \sigma_{x}^{d}>\right] \delta(z-x)+\frac{1}{g}\left[\frac{\nabla_{i x}}{\left(-\nabla_{x}^{2}\right)}<\imath \Phi_{\tau(l) v}^{f} \imath \Phi_{\lambda(k) w}^{e} \imath A_{i x}^{a}>\right]<\imath \bar{c}_{x}^{a} c_{z}^{d}>\right. \\
& +\frac{1}{g}\left[\frac{\nabla_{i x}}{\left(-\nabla_{x}^{2}\right)}<\imath \Phi_{\lambda(k) w}^{e} \imath A_{i x}^{a}>\right]<\imath \bar{c}_{x}^{a} \imath c_{z}^{d} \imath \Phi_{\tau(l) v}^{f}>+\frac{1}{g}\left[\frac{\nabla_{i x}}{\left(-\nabla_{x}^{2}\right)}<\imath \Phi_{\tau(l) v}^{f} \imath A_{i x}^{a}>\right]<\imath \bar{c}_{x}^{a} \imath c_{z}^{d} \imath \Phi_{\lambda(k) w}^{e}> \\
& -<\imath \Phi_{\lambda(k) w}^{e} \imath \sigma_{x}^{a}>\left[f^{a b c} \frac{\delta^{2}}{\delta \imath \Phi_{\tau(l) v}^{f} \delta \imath c_{z}^{d}}<\imath \rho_{x}^{b} \bar{\eta}_{x}^{c}>\left.\right|_{J=0}-f^{a f d} \delta_{\sigma \tau} \delta(v-x) \delta(z-x)\right] \\
& -<\imath \Phi_{\tau(l) v}^{f} \imath \sigma_{x}^{a}>\left[f^{a b c} \frac{\delta^{2}}{\delta \imath \Phi_{\lambda(k) w}^{f} \delta \imath c_{z}^{d}}<\imath \rho_{x}^{b} \bar{\eta}_{x}^{c}>\left.\right|_{J=0}-f^{a e d} \delta_{\sigma \lambda} \delta(w-x) \delta(z-x)\right] \\
& -<\imath \Phi_{\lambda(k) w}^{e} \imath A_{i x}^{a}>t_{i j}(\vec{x})\left[f^{a b c} \frac{\delta^{2}}{\delta \imath \Phi_{\tau(l) v}^{f} \delta \imath c_{z}^{d}}<\imath J_{j x}^{b} \imath \bar{\eta}_{x}^{c}>\left.\right|_{J=0}-f^{a f d} \delta_{j l} \delta_{A \tau} \delta(v-x) \delta(z-x)\right] \\
& \left.-<\imath \Phi_{\tau(l) v}^{f} \imath A_{i x}^{a}>t_{i j}(\vec{x})\left[f^{a b c} \frac{\delta^{2}}{\delta \imath \Phi_{\lambda(k) w}^{e} \delta \imath c_{z}^{d}}<\imath J_{j x}^{b} \imath \bar{\eta}_{x}^{c}>\left.\right|_{J=0}-f^{a e d} \delta_{j k} \delta_{A \lambda} \delta(w-x) \delta(z-x)\right]\right\} .
\end{aligned}
$$

In the above, we have made use of the results Eqs. (3.9) and (3.13) to eliminate such terms. Because the indices $\lambda$ and $\tau$ refer to field types that commute, the above equation actually represents three separate equations. Let us define

$$
\begin{gathered}
\tilde{\Gamma}_{\sigma ; \bar{c} c \tau(l)}^{a d f}(x, z, v)=g f^{a b c} \frac{\delta^{2}}{\delta \imath \Phi_{\tau(l) v}^{f} \delta \imath c_{z}^{d}}<\imath \rho_{x}^{b} \imath \bar{\eta}_{x}^{c}>\left.\right|_{J=0}, \\
\tilde{\Gamma}_{A ; \bar{c} c \tau j(l)}^{a d f}(x, z, v)=g f^{a b c} \frac{\delta^{2}}{\delta \imath \Phi_{\tau(l) v}^{f} \delta \imath c_{z}^{d}}<\imath J_{j x}^{b} \imath \bar{\eta}_{x}^{c}>\left.\right|_{J=0},
\end{gathered}
$$

(these expressions will be explained later). We further notice that by taking two functional derivatives of Eq. (2.18) and setting sources to zero then we have

$$
<\imath \bar{c}_{x}^{a} \imath c_{z}^{d} \imath \Phi_{\tau(l) v}^{f}>=-g f^{a d f} \delta_{A \tau} \delta_{j l} \nabla_{j x} \delta(v-x) \delta(z-x)-\nabla_{j x} \tilde{\Gamma}_{A ; \bar{c} c \tau j(l)}^{a d f}(x, z, v) .
$$

This equation is of course the precursor to the Dyson-Schwinger equation for the temporal and spatial ghost-gluon vertices. Our identities can thus be written

$$
\begin{aligned}
0= & \int d^{4} x \delta\left(t-x_{0}\right)\left\{-\imath\left[\partial_{x}^{0}<\imath \Phi_{\tau(l) v}^{f} \imath \Phi_{\lambda(k) w}^{e} \imath \sigma_{x}^{d}>\right] \delta(z-x)+\left[\frac{\nabla_{i x}}{\left(-\nabla_{x}^{2}\right)}<\imath \Phi_{\tau(l) v}^{f} \imath \Phi_{\lambda(k) w}^{e} \imath A_{i x}^{a}>\right]<\imath \bar{c}_{x}^{a} \imath c_{z}^{d}>\right. \\
& -<\imath \Phi_{\lambda(k) w}^{e} \imath A_{i x}^{a}>\left[\tilde{\Gamma}_{A ; \bar{c} c \tau i(l)}^{a d f}(x, z, v)+g f^{a d f} \delta_{i l} \delta_{A \tau} \delta(v-x) \delta(z-x)\right] \\
& -<\imath \Phi_{\tau(l) v}^{f} \imath A_{i x}^{a}>\left[\tilde{\Gamma}_{A ; \bar{c} c \lambda i(k)}^{a d e}(x, z, w)+g f^{a d e} \delta_{i k} \delta_{A \lambda} \delta(w-x) \delta(z-x)\right]
\end{aligned}
$$




$$
\begin{aligned}
& -<\imath \Phi_{\lambda(k) w}^{e} \imath \sigma_{x}^{a}>\left[\tilde{\Gamma}_{\sigma ; \bar{c} c \tau(l)}^{a d f}(x, z, v)+g f^{a d f} \delta_{\sigma \tau} \delta(v-x) \delta(z-x)\right] \\
& \left.-<\imath \Phi_{\tau(l) v}^{f} \imath \sigma_{x}^{a}>\left[\tilde{\Gamma}_{\sigma ; \bar{c} c \lambda(k)}^{a d e}(x, z, w)+g f^{a d e} \delta_{\sigma \lambda} \delta(w-x) \delta(z-x)\right]\right\} .
\end{aligned}
$$

Let us now introduce our convention for the Fourier transform of the three-point functions (similarly for higher $n$-point functions):

$$
f(x, y, z)=\int d k_{1} d k_{2} d k_{3}(2 \pi)^{4} \delta\left(k_{1}+k_{2}+k_{3}\right) e^{-\imath k_{1} \cdot x-\imath k_{2} \cdot y-\imath k_{3} \cdot z} f\left(k_{1}, k_{2}, k_{3}\right) .
$$

All momenta of the three-point functions are defined as incoming and for brevity we do not split the temporal and spatial sets of arguments. Returning to Eq. (4.4), after some manipulation we arrive at the three Slavnov-Taylor identities $\left(k_{1}+k_{2}+k_{3}=0\right)$ :

$$
\begin{aligned}
k_{3}^{0} \Gamma_{\tau \lambda \sigma(l k)}^{f e d}\left(k_{1}, k_{2}, k_{3}\right)= & \imath \frac{k_{3 i}}{\vec{k}_{3}^{2}} \Gamma_{\tau \lambda A(l k) i}^{f e a}\left(k_{1}, k_{2}, k_{3}\right) \Gamma_{\overline{c c}}^{a d}\left(-\vec{k}_{3}\right) \\
& -\Gamma_{\lambda A(k) i}^{e a}\left(k_{2}\right)\left[\tilde{\Gamma}_{A ; \bar{c} c \tau i(l)}^{a d f}\left(k_{2}+q_{0}, k_{3}-q_{0}, k_{1}\right)+g f^{a d f} \delta_{i l} \delta_{A \tau}\right] \\
& -\Gamma_{\tau A(l) i}^{f a}\left(k_{1}\right)\left[\tilde{\Gamma}_{A ; \bar{c} c \lambda i(k)}^{a d e}\left(k_{1}+q_{0}, k_{3}-q_{0}, k_{2}\right)+g f^{a d e} \delta_{i k} \delta_{A \lambda}\right] \\
& -\Gamma_{\lambda \sigma(k)}^{e a}\left(k_{2}\right)\left[\tilde{\Gamma}_{\sigma ; \bar{c} c \tau(l)}^{a d f}\left(k_{2}+q_{0}, k_{3}-q_{0}, k_{1}\right)+g f^{a d f} \delta_{\sigma \tau}\right] \\
& -\Gamma_{\tau \sigma(l)}^{f a}\left(k_{1}\right)\left[\tilde{\Gamma}_{\sigma ; \bar{c} c \lambda(k)}^{a d e}\left(k_{1}+q_{0}, k_{3}-q_{0}, k_{2}\right)+g f^{a d e} \delta_{\sigma \lambda}\right] .
\end{aligned}
$$

These identities can easily be verified at tree-level, using the Feynman rules presented in Ref. [28] (and for completeness presented in Appendix A). They are the Coulomb gauge analogue of the familiar Slavnov-Taylor identity for the three-gluon vertex [34]. As in the previous section, the energy $q_{0}$ is injected into the ghost line of the various factors but one cannot cancel terms using symmetry properties anymore (as was the case for the $\tilde{\Sigma}$ kernels) because of the presence of other energy arguments in the equations. Neglecting the $\tilde{\Gamma}$ terms, we can see that each vertex involving a functional derivative with respect to the temporal $\sigma$-field is fully determined (again because $k_{3}^{0}$ is a scalar quantity) given the corresponding (spatially contracted) vertex involving the derivative with respect to the spatial $A$-field.

Let us now discuss the form of the $\tilde{\Gamma}$ factors. From the definition, Eq. (4.2), and taking functional derivatives in standard fashion, we have

$$
\begin{aligned}
\tilde{\Gamma}_{\sigma ; \bar{c} c \tau}^{a d f}(x, z, v)= & g f^{a b c} \frac{\delta^{2}}{\delta \imath \Phi_{\tau v}^{f} \delta \imath c_{z}^{d}}<\imath \rho_{x}^{b} \imath \bar{\eta}_{x}^{c}>\left.\right|_{J=0} \\
= & g f^{a b c}\left\{<\imath \rho_{x}^{b} \imath J_{\varepsilon}><\imath \Phi_{\varepsilon} \imath \Phi_{\tau v}^{f} \imath \Phi_{\lambda}><\imath J_{\lambda} \imath J_{\kappa}><\imath \bar{\eta}_{x}^{c} \imath \eta_{u}^{g}><\imath \bar{c}_{u}^{g} \imath c_{z}^{d} \imath \Phi_{\kappa}>\right. \\
& -<\imath \rho_{x}^{b} \imath J_{\kappa}><\imath \bar{\eta}_{x}^{c} \imath \eta_{u}^{g}><\bar{c}_{u}^{g} \imath c_{z}^{d} \imath \Phi_{\kappa} \imath \Phi_{\tau(l) v}^{f}> \\
& \left.+<\imath \rho_{x}^{b} \imath J_{\kappa}><\imath \bar{\eta}_{x}^{c} \imath \eta_{u}^{g}><\imath \bar{c}_{u}^{g} \imath c_{s}^{h} \imath \Phi_{\tau v}^{f}><\imath \bar{\eta}_{s}^{h} \eta_{r}^{i}><\imath \bar{c}_{r}^{i} \imath c_{z}^{d} \imath \Phi_{\kappa}>\right\}
\end{aligned}
$$

where we recall that having explicitly extracted the Grassmann-valued fields, the repeated indices refer here to only the $\sigma$ or $A$-fields. The coordinates $u, s$ and $r$ are implicitly integrated over. We further omit the possible spatial index (l) associated when $\tau$ refers to the $\vec{A}$-field for notational convenience. A similar expression exists for $\tilde{\Gamma}_{A ; \bar{c} c \tau}$. In momentum space, we have $\left(p_{1}+p_{2}+p_{3}=0\right)$

$$
\begin{aligned}
\tilde{\Gamma}_{\sigma ; \bar{c} c \tau}^{a d f}\left(p_{1}, p_{2}, p_{3}\right)= & g f^{a b c} \int d k W_{\sigma \varepsilon}^{b g}(k) \Gamma_{\varepsilon \tau \mu}^{g f h}\left(k, p_{3},-k-p_{3}\right) W_{\mu \kappa}^{h i}\left(k+p_{3}\right) W_{\bar{c} c}^{c j}\left(p_{1}-k\right) \Gamma_{\bar{c} c \kappa}^{j d i}\left(p_{1}-k, p_{2}, k+p_{3}\right) \\
& -g f^{a b c} \int d k W_{\sigma \kappa}^{b g}(k) W_{\bar{c} c}^{c h}\left(p_{1}-k\right) \Gamma_{\bar{c} c \kappa \tau}^{h d g f}\left(p_{1}-k, p_{2}, k, p_{3}\right) \\
& +g f^{a b c} \int d k W_{\sigma \kappa}^{b g}(k) W_{\bar{c} c}^{c h}\left(p_{1}-k\right) \Gamma_{\bar{c} c \tau}^{h i f}\left(p_{1}-k, p_{2}+k, p_{3}\right) W_{\bar{c} c}^{i j}\left(-p_{2}-k\right) \Gamma_{\bar{c} c \kappa}^{j d g}\left(-p_{2}-k, p_{2}, k\right),
\end{aligned}
$$

(we make the internal color indices explicit) again with similar expressions for the other $\tilde{\Gamma}$ factors. Given that there is the implicit summation over $\varepsilon, \mu$ and $\kappa$ (referring to the field types $\sigma$ and $A$ ), what this means is that the $\tilde{\Gamma}$ considered 
so far are in general dependent not only on the non-ghost temporal three-point functions that are explicit in Eq. (4.6) (thus showing that Eq. (4.6) actually forms a set of nonlinear integral equations) but on the further temporal Green's functions $\Gamma_{\bar{c} c \sigma}, \Gamma_{\bar{c} c \sigma A}$ and $\Gamma_{\bar{c} c \sigma \sigma}$ (and of course all the other two-point functions, the purely spatial $A$ and the ghost three- and four-point functions).

\section{B. One pair of further ghost derivatives}

Let us now consider the Slavnov-Taylor identities that arise if we functionally differentiate Eq. (2.26) with respect to at least $\imath \bar{c}_{v}^{f}$ and $\imath c_{w}^{e}$. If we restrict ourselves to considering no further ghost/antighost functional derivatives, then we are able to set the corresponding fields/sources to zero whilst maintaining the rest without confusion. The resulting expression reads:

$$
\begin{aligned}
0= & \int d^{4} x \delta\left(t-x_{0}\right)\left\{\frac{\imath}{g}\left[\partial_{x}^{0}<\imath \bar{c}_{v}^{f} \imath c_{z}^{d} \imath \sigma_{x}^{e}>\right] \delta(w-x)-\frac{\imath}{g}\left[\partial_{x}^{0}<\imath \bar{c}_{v}^{f} \imath c_{w}^{e} \imath \sigma_{x}^{d}>\right] \delta(z-x)\right. \\
& -\frac{1}{g}\left[\frac{\nabla_{i x}}{\left(-\nabla_{x}^{2}\right)}<\imath \bar{c}_{v}^{f} \imath c_{z}^{d} \imath A_{i x}^{a}>\right]<\imath \bar{c}_{x}^{a} \imath c_{w}^{e}>+\frac{1}{g}\left[\frac{\nabla_{i x}}{\left(-\nabla_{x}^{2}\right)}<\imath \bar{c}_{v}^{f} \imath c_{w}^{e} \imath A_{i x}^{a}>\right]<\imath \bar{c}_{x}^{a} \imath c_{z}^{d}> \\
& +\frac{1}{g}\left[\frac{\nabla_{i x}}{\left(-\nabla_{x}^{2}\right)}<\imath A_{i x}^{a}>\right]<\imath \bar{c}_{v}^{f} \imath c_{w}^{e} \imath \bar{c}_{x}^{a} \imath c_{z}^{d}> \\
& +f^{a b c}<\imath \bar{c}_{v}^{f} \imath c_{z}^{d} \imath \sigma_{x}^{a}>\left[\frac{\delta}{\delta \imath c_{w}^{e}}<\imath \rho_{x}^{b} \imath \bar{\eta}_{x}^{c}>-\imath \sigma_{x}^{b} \delta^{e c} \delta(w-x)\right]-f^{a b c}<\imath \bar{c}_{v}^{f} \imath c_{w}^{e} \imath \sigma_{x}^{a}>\left[\frac{\delta}{\delta \imath c_{z}^{d}}<\imath \rho_{x}^{b} \imath \bar{\eta}_{x}^{c}>-\imath \sigma_{x}^{b} \delta^{d c} \delta(z-x)\right] \\
& -f^{a b c}<\imath \sigma_{x}^{a}>\frac{\delta^{3}}{\delta \imath \bar{c}_{v}^{f} \delta \imath c_{w}^{e} \delta \imath c_{z}^{d}}<\imath \rho_{x}^{b} \imath \bar{\eta}_{x}^{c}>+f^{a b c}<\imath \bar{c}_{v}^{f} \imath c_{z}^{d} \imath A_{i x}^{a}>t_{i j}(\vec{x})\left[\frac{\delta}{\delta \imath c_{w}^{e}}<\imath J_{j x}^{b} \imath \bar{\eta}_{x}^{c}>-\imath A_{j x}^{b} \delta^{e c} \delta(w-x)\right] \\
& -f^{a b c}<\imath \bar{c}_{v}^{f} \imath c_{w}^{e} \imath A_{i x}^{a}>t_{i j}(\vec{x})\left[\frac{\delta}{\delta \imath c_{z}^{d}}<\imath J_{j x}^{b} \imath \bar{\eta}_{x}^{c}>-\imath A_{j x}^{b} \delta^{d c} \delta(z-x)\right]-f^{a b c}<\imath A_{i x}^{a}>t_{i j}(\vec{x}) \frac{\delta^{3}}{\delta \imath \bar{c}_{v}^{f} \delta \imath c_{w}^{e} \delta \imath c_{z}^{d}}<\imath J_{j x}^{b} \imath \bar{\eta}_{x}^{c}> \\
& \left.+\frac{1}{2} f^{a b c}<\bar{c}_{v}^{f} \imath c_{x}^{a}>\left[\frac{\delta^{2}}{\delta \imath c_{w}^{e} \delta \imath c_{z}^{d}}<\imath \bar{\eta}_{x}^{b} \bar{\eta}_{x}^{c}>-2 \delta^{d b} \delta^{e c} \delta(z-x) \delta(w-x)\right]\right\}_{\bar{\eta}=\eta=0} .
\end{aligned}
$$

To derive the identity for the ghost-gluon vertex, we further set the remaining sources to zero (we will return to Eq. (4.9) later in this subsection to derive more identities) and again using the results given by Eqs. (3.9) and (3.13) we get

$$
\begin{aligned}
0= & \int d^{4} x \delta\left(t-x_{0}\right)\left\{\frac{\imath}{g}\left[\partial_{x}^{0}<\imath \bar{c}_{v}^{f} \imath c_{z}^{d} \imath \sigma_{x}^{e}>\right] \delta(w-x)-\frac{\imath}{g}\left[\partial_{x}^{0}<\imath \bar{c}_{v}^{f} \imath c_{w}^{e} \imath \sigma_{x}^{d}>\right] \delta(z-x)\right. \\
& -\frac{1}{g}\left[\frac{\nabla_{i x}}{\left(-\nabla_{x}^{2}\right)}<\imath \bar{c}_{v}^{f} \imath c_{z}^{d} \imath A_{i x}^{a}>\right]<\imath \bar{c}_{x}^{a} \imath c_{w}^{e}>+\frac{1}{g}\left[\frac{\nabla_{i x}}{\left(-\nabla_{x}^{2}\right)}<\imath \bar{c}_{v}^{f} \imath c_{w}^{e} \imath A_{i x}^{a}>\right]<\imath \bar{c}_{x}^{a} \imath c_{z}^{d}> \\
& \left.+\frac{1}{2 g}<\imath \bar{c}_{v}^{f} \imath c_{x}^{a}>\tilde{\Gamma}_{\bar{c} ; \bar{c} c c}^{a d e}(x, z, w)-f^{a d e}<\imath \bar{c}_{v}^{f} \imath c_{x}^{a}>\delta(z-x) \delta(w-x)\right\} .
\end{aligned}
$$

In the above, we have defined

$$
\begin{aligned}
\tilde{\Gamma}_{\bar{c} ; \bar{c} c c}^{a d e}(x, z, w) & =g f^{a b c} \frac{\delta^{2}}{\delta \imath c_{w}^{e} \delta \imath c_{z}^{d}}<\imath \bar{\eta}_{x}^{b} \imath \bar{\eta}_{x}^{c}>\left.\right|_{J=0} \\
& =g f^{a b c}<\imath \bar{\eta}_{x}^{b} \imath \eta_{\nu}><\imath \bar{\eta}_{x}^{c} \imath \eta_{\mu}>\left[2<\imath J_{\kappa} \imath J_{\varepsilon}><\imath \bar{c}_{\nu} \imath c_{z}^{d} \imath \Phi_{\kappa}><\imath \bar{c}_{\mu} \imath c_{w}^{e} \imath \Phi_{\varepsilon}>-<\imath \bar{c}_{\nu} \imath c_{w}^{e} \imath c_{z}^{d} l \bar{c}_{\mu}>\right]
\end{aligned}
$$

(internal indices $\kappa$ and $\varepsilon$ referring only to $A$ or $\sigma$-fields as in the previous subsection). Considering now the Fourier transform and using the same conventions as before, we arrive at the Slavnov-Taylor identity for the temporal ghostgluon vertex

$$
\begin{aligned}
0= & k_{3}^{0} \Gamma_{\bar{c} c \sigma}^{f d e}\left(k_{1}, k_{2}, k_{3}\right)-\frac{\imath k_{3 i}}{\vec{k}_{3}^{2}} \Gamma_{\bar{c} c A i}^{f d a}\left(k_{1}, k_{2}, k_{3}\right) \Gamma_{\bar{c} c}^{a e}\left(-\vec{k}_{3}\right) \\
& -\left(k_{2}^{0}+q_{0}\right) \Gamma_{\bar{c} c \sigma}^{f e d}\left(k_{1}, k_{3}-q_{0}, k_{2}+q_{0}\right)+\frac{\imath k_{2 i}}{\vec{k}_{2}^{2}} \Gamma_{\bar{c} c A i}^{f e a}\left(k_{1}, k_{3}-q_{0}, k_{2}+q_{0}\right) \Gamma_{\bar{c} c}^{a d}\left(-\vec{k}_{2}\right) \\
& +\frac{1}{2} \Gamma_{\bar{c} c}^{f a}\left(\vec{k}_{1}\right) \tilde{\Gamma}_{\bar{c} ; \bar{c} c c}^{a d e}\left(k_{1}+q_{0}, k_{2}, k_{3}-q_{0}\right)-g f^{a d e} \Gamma_{\bar{c} c}^{f a}\left(\vec{k}_{1}\right)
\end{aligned}
$$


with

$$
\begin{aligned}
\tilde{\Gamma}_{\bar{c} ; \bar{c} c c}^{a d e}\left(p_{1}, p_{2}, p_{3}\right)=g f^{a b c} \int d k W_{\bar{c} c}^{b f}\left(p_{1}-k\right) W_{\bar{c} c}^{c g}(k) & \left\{2 \Gamma_{\bar{c} c \kappa}^{f d h}\left(p_{1}-k, p_{2}, p_{3}+k\right) \Gamma_{\bar{c} c \varepsilon}^{g e i}\left(k, p_{3},-p_{3}-k\right) W_{\kappa \varepsilon}^{h i}\left(-p_{3}-k\right)\right. \\
& \left.-\Gamma_{\bar{c} c c \bar{c}}^{f e d g}\left(p_{1}-k, p_{3}, p_{2}, k\right)\right\}
\end{aligned}
$$

Recall that we are free to choose the energy injection, $q_{0}$, at will. If we set $k_{2}^{0}+q_{0}=0$ in Eq. (4.12), then (given that for such general spacelike momentum configurations, the Green's functions can have no singularities) the $\Gamma_{\bar{c}} c \sigma$ term of the middle line drops out and we have an unambiguous nonlinear integral equation for $\Gamma_{\bar{c} c \sigma}$. Crucially, as far as the three-point functions involving the temporal $\sigma$-field are concerned, the equation forms a closed expression without approximation. One can immediately verify this identity at tree-level using the Feynman rules of Ref. [28] (and summarized in Appendix A). A similar Slavnov-Taylor identity for the ghost-gluon vertex in Landau gauge does in fact exist $[6,12]$.

Returning to Eq. (4.9), we take the further functional derivative with respect to $\imath \Phi_{\lambda(k) u}^{g}$ (again the field type $\lambda$ refers only to either the spatial $A$-field with index $k$ or to the temporal $\sigma$-field) and set sources to zero. Making use of the results Eqs. (3.9) and (3.13) to eliminate such terms and if we further use the appropriate functional derivatives of Eq. (2.18) as before, then we have Eq. (B.1) (such lengthy configuration space expressions are relegated to Appendix B). Equation (B.1) involves three new kernels: $\tilde{\Gamma}_{\sigma ; \bar{c} c c \bar{c}}$ and $\tilde{\Gamma}_{\bar{c} ; \bar{c} c c \lambda}$ are given by Eqs. (B.2) and (B.3), respectively; the third kernel, $\tilde{\Gamma}_{A ; \bar{c} c c \bar{c}}$, has a similar expression to Eq. (B.2). There is only one possible new Green's function involving derivatives with respect to the $\sigma$-field and this is $\Gamma_{\bar{c} c \bar{c} c \sigma}$. Taking the Fourier transform of Eq. (B.1) leads to the following Slavnov-Taylor identity in momentum space $\left(\sum k_{a}=0\right)$ :

$$
\begin{aligned}
0= & k_{4}^{0} \Gamma_{\bar{c} c \lambda \sigma(k)}^{f d g e}\left(k_{1}, k_{2}, k_{3}, k_{4}\right)-\frac{\imath k_{4 i}}{\vec{k}_{4}^{2}} \Gamma_{\bar{c} c \lambda A(k) i}^{f d g a}\left(k_{1}, k_{2}, k_{3}, k_{4}\right) \Gamma_{\bar{c} c}^{a e}\left(-\vec{k}_{4}\right) \\
& -\left(k_{2}^{0}+q_{0}\right) \Gamma_{\bar{c} c \lambda \sigma(k)}^{f e g d}\left(k_{1}, k_{4}-q_{0}, k_{3}, k_{2}+q_{0}\right)+\frac{\imath k_{2 i}}{\vec{k}_{2}^{2}} \Gamma_{\bar{c} c \lambda A(k) i}^{f e g a}\left(k_{1}, k_{4}-q_{0}, k_{3}, k_{2}+q_{0}\right) \Gamma_{\bar{c} c}^{a d}\left(-\vec{k}_{2}\right) \\
& +\Gamma_{\bar{c} c \sigma}^{f d a}\left(k_{1}, k_{2}, k_{3}+k_{4}\right)\left[\tilde{\Gamma}_{\sigma ; \bar{c} c \lambda(k)}^{a e g}\left(q_{0}-k_{3}-k_{4}, k_{4}-q_{0}, k_{3}\right)-g f^{a g e} \delta_{\sigma \lambda}\right] \\
& +\Gamma_{\bar{c} c A i}^{f d a}\left(k_{1}, k_{2}, k_{3}+k_{4}\right)\left[\tilde{\Gamma}_{A ; \bar{c} c \lambda i(k)}^{a e g}\left(q_{0}-k_{3}-k_{4}, k_{4}-q_{0}, k_{3}\right)-g f^{a g e} \delta_{k i} \delta_{A \lambda}\right] \\
& -\Gamma_{\bar{c} c \sigma}^{f e a}\left(k_{1}, k_{4}-q_{0}, k_{2}+k_{3}+q_{0}\right)\left[\tilde{\Gamma}_{\sigma ; \bar{c} c \lambda(k)}^{a d g}\left(-k_{2}-k_{3}, k_{2}, k_{3}\right)-g f^{a g d} \delta_{\sigma \lambda}\right] \\
& -\Gamma_{\bar{c} c A i}^{f e a}\left(k_{1}, k_{4}-q_{0}, k_{2}+k_{3}+q_{0}\right)\left[\tilde{\Gamma}_{A ; \bar{c} c \lambda i(k)}^{a d g}\left(-k_{2}-k_{3}, k_{2}, k_{3}\right)-g f^{a g d} \delta_{k i} \delta_{A \lambda}\right] \\
& -\Gamma_{\lambda \sigma(k)}^{g a}\left(k_{3}\right) \tilde{\Gamma}_{\sigma ; \bar{c} c c \bar{c}}^{a d e f}\left(k_{3}+q_{0}, k_{2}, k_{4}-q_{0}, k_{1}\right)-\Gamma_{\lambda A(k) i}^{g a}\left(k_{3}\right) \tilde{\Gamma}_{A ; \bar{c} c c \bar{c} i}^{a d e f}\left(k_{3}+q_{0}, k_{2}, k_{4}-q_{0}, k_{1}\right) \\
& +\frac{1}{2} \Gamma_{\bar{c} c \lambda(k)}^{f a g}\left(k_{1}, k_{2}+k_{4}, k_{3}\right)\left[\tilde{\Gamma}_{\bar{c} ; \bar{c} c c}^{a d e}\left(q_{0}-k_{2}-k_{4}, k_{2}, k_{4}-q_{0}\right)-2 g f^{a d e}\right] \\
& +\frac{1}{2} \Gamma_{\bar{c} c}^{f a}\left(\vec{k}_{1}\right) \tilde{\Gamma}_{\bar{c} ; \bar{c} c c \lambda(k)}^{a d e g}\left(k_{1}+q_{0}, k_{2}, k_{4}-q_{0}, k_{3}\right),
\end{aligned}
$$

with the kernels (because of the proliferation of color indices, we resort to Greek superscripts)

$$
\begin{aligned}
& \tilde{\Gamma}_{\sigma ; \bar{c} c c \bar{c}}^{a d e f}\left(p_{1}, p_{2}, p_{3}, p_{4}\right)=g f^{a b c} \int d k W_{\sigma \nu}^{b \nu}(k) W_{\bar{c} c}^{c \gamma}\left(p_{1}-k\right) \times \\
& \quad\left\{\Gamma _ { \overline { c } c \nu } ^ { f \mu \nu } ( p _ { 4 } , - p _ { 4 } - k , k ) W _ { \overline { c } c } ^ { \mu \varepsilon } ( p _ { 4 } + k ) \left[\Gamma_{\bar{c} c \alpha}^{\varepsilon d \alpha}\left(p_{4}+k, p_{2},-k-p_{2}-p_{4}\right) W_{\alpha \kappa}^{\alpha \kappa}\left(k+p_{2}+p_{4}\right) \Gamma_{\bar{c} c \kappa}^{\gamma e \kappa}\left(p_{1}-k, p_{3}, k+p_{2}+p_{4}\right)\right.\right. \\
& \left.\quad-\Gamma_{\bar{c} c \alpha}^{\varepsilon e \alpha}\left(p_{4}+k, p_{3}, p_{1}+p_{2}-k\right) W_{\alpha \kappa}^{\alpha \kappa}\left(k-p_{1}-p_{2}\right) \Gamma_{\bar{c} c \kappa}^{\gamma d \kappa}\left(p_{1}-k, p_{2}, k-p_{1}-p_{2}\right)\right] \\
& \quad+\Gamma_{\bar{c} c \varepsilon}^{f \mu \varepsilon}\left(p_{4}, k+p_{2}, p_{1}+p_{3}-k\right) W_{\bar{c} c}^{\mu \alpha}\left(-p_{2}-k\right) \Gamma_{\bar{c} c \nu}^{\alpha d \nu}\left(-p_{2}-k, p_{2}, k\right) W_{\varepsilon \kappa}^{\varepsilon \kappa}\left(k+p_{2}+p_{4}\right) \Gamma_{\bar{c} c \kappa}^{\gamma e \kappa}\left(p_{1}-k, p_{3}, k+p_{2}+p_{4}\right) \\
& \quad-\Gamma_{\bar{c} c \varepsilon}^{f \mu \varepsilon}\left(p_{4}, k+p_{3}, p_{1}+p_{2}-k\right) W_{\bar{c} c}^{\mu \alpha}\left(-p_{3}-k\right) \Gamma_{\bar{c} c \nu}^{\alpha e \nu}\left(-p_{3}-k, p_{3}, k\right) W_{\varepsilon \kappa}^{\varepsilon \kappa}\left(k+p_{3}+p_{4}\right) \Gamma_{\bar{c} c \kappa}^{\gamma d \kappa}\left(p_{1}-k, p_{2}, k+p_{3}+p_{4}\right) \\
& \quad+\Gamma_{\bar{c} c \alpha \nu}^{f e \alpha \nu}\left(p_{4}, p_{3},-k-p_{3}-p_{4}, k\right) W_{\alpha \kappa}^{\alpha \kappa}\left(k+p_{3}+p_{4}\right) \Gamma_{\bar{c} c \kappa}^{\gamma d \kappa}\left(p_{1}-k, p_{2}, k+p_{3}+p_{4}\right) \\
& \quad-\Gamma_{\bar{c} c \alpha \nu}^{f d \alpha \nu}\left(p_{4}, p_{2},-k-p_{2}-p_{4}, k\right) W_{\alpha \kappa}^{\alpha \kappa}\left(k+p_{2}+p_{4}\right) \Gamma_{\bar{c} c \kappa}^{\gamma e \kappa}\left(p_{1}-k, p_{3}, k+p_{2}+p_{4}\right) \\
& \quad+\Gamma_{\bar{c} c \bar{c} c}^{f \kappa \gamma d}\left(p_{4}, k+p_{3}, p_{1}-k, p_{2}\right) W_{\bar{c} c}^{\kappa \alpha}\left(-p_{3}-k\right) \Gamma_{\bar{c} c \nu}^{\alpha e \nu}\left(-p_{3}-k, p_{3}, k\right) \\
& \quad-\Gamma_{\bar{c} c \bar{c} c}^{f \kappa \gamma e}\left(p_{4}, k+p_{2}, p_{1}-k, p_{3}\right) W_{\bar{c} c}^{\kappa \alpha}\left(-p_{2}-k\right) \Gamma_{\bar{c} c \nu}^{\alpha d \nu}\left(-p_{2}-k, p_{2}, k\right) \\
& \left.\quad+\Gamma_{\bar{c} c \nu}^{f \mu \nu}\left(p_{4},-p_{4}-k, k\right) W_{\bar{c} c}^{\mu \kappa}\left(p_{4}+k\right) \Gamma_{\bar{c} c \bar{c} c}^{\kappa e \gamma d}\left(p_{4}+k, p_{3}, p_{1}-k, p_{2}\right)-\Gamma_{\bar{c} c \bar{c} c \nu}^{f e \gamma d \nu}\left(p_{4}, p_{3}, p_{1}-k, p_{2}, k\right)\right\}
\end{aligned}
$$




$$
\begin{aligned}
\tilde{\Gamma}_{\bar{c} ; \bar{c} c c \sigma}^{a d e g}\left(p_{1}, p_{2}, p_{3}, p_{4}\right)=g f^{a b c} \int d k W_{\bar{c} c}^{b \nu}(k) W_{\bar{c} c}^{c \gamma}\left(p_{1}-k\right) \times \\
\quad\left\{2 \Gamma_{\bar{c} c \sigma}^{\nu \mu g}\left(k,-p_{4}-k, p_{4}\right) W_{\bar{c} c}^{\mu \varepsilon}\left(p_{4}+k\right) \Gamma_{\bar{c} c \alpha}^{\varepsilon e \alpha}\left(p_{4}+k, p_{3}, p_{1}+p_{2}-k\right) W_{\alpha \kappa}^{\alpha \kappa}\left(k-p_{1}-p_{2}\right) \Gamma_{\bar{c} c \kappa}^{\gamma d \kappa}\left(p_{1}-k, p_{2}, k-p_{1}-p_{2}\right)\right. \\
\quad-2 \Gamma_{\bar{c} c \sigma}^{\nu \mu g}\left(k,-p_{4}-k, p_{4}\right) W_{\bar{c} c}^{\mu \varepsilon}\left(p_{4}+k\right) \Gamma_{\bar{c} c \alpha}^{\varepsilon d \alpha}\left(p_{4}+k, p_{2}, p_{1}+p_{3}-k\right) W_{\alpha \kappa}^{\alpha \kappa}\left(k-p_{1}-p_{3}\right) \Gamma_{\bar{c} c \kappa}^{\gamma e \kappa}\left(p_{1}-k, p_{3}, k-p_{1}-p_{3}\right) \\
\quad-2 \Gamma_{\bar{c} c \varepsilon \sigma}^{\nu e \varepsilon g}\left(k, p_{3},-p_{3}-p_{4}-k, p_{4}\right) \Gamma_{\bar{c} c \kappa}^{\gamma d \kappa}\left(p_{1}-k, p_{2}, k+p_{3}+p_{4}\right) W_{\varepsilon \kappa}^{\varepsilon \kappa}\left(k+p_{3}+p_{4}\right) \\
\quad+2 \Gamma_{\bar{c} c \varepsilon \sigma}^{\nu d \varepsilon g}\left(k, p_{2},-p_{2}-p_{4}-k, p_{4}\right) \Gamma_{\bar{c} c \kappa}^{\gamma e \kappa}\left(p_{1}-k, p_{3}, k+p_{2}+p_{4}\right) W_{\varepsilon \kappa}^{\varepsilon \kappa}\left(k+p_{2}+p_{4}\right) \\
\quad+2 \Gamma_{\bar{c} c \varepsilon}^{\nu e \varepsilon}\left(k, p_{3},-p_{3}-k\right) W_{\varepsilon \mu}^{\varepsilon \mu}\left(p_{3}+k\right) \Gamma_{\mu \sigma \alpha}^{\mu g \alpha}\left(p_{3}+k, p_{4}, p_{1}+p_{2}-k\right) W_{\alpha \kappa}^{\alpha \kappa}\left(k-p_{1}-p_{2}\right) \Gamma_{\bar{c} c \kappa}^{\gamma d \kappa}\left(p_{1}-k, p_{2}, k-p_{1}-p_{2}\right) \\
\left.\quad-2 \Gamma_{\bar{c} c \sigma}^{\nu \varepsilon g}\left(k,-p_{4}-k, p_{4}\right) W_{\bar{c} c}^{\varepsilon \kappa}\left(p_{4}+k\right) \Gamma_{\bar{c} c \bar{c} c}^{\kappa e \gamma d}\left(p_{4}+k, p_{3}, p_{1}-k, p_{2}\right)+\Gamma_{\bar{c} c \bar{c} c \sigma}^{\nu e \gamma d g}\left(k, p_{3}, p_{1}-k, p_{2}, p_{4}\right)\right\} .
\end{aligned}
$$

(A similar expression exists for the kernel $\tilde{\Gamma}_{A ; \bar{c} c c \bar{c}}$.) As before, since we may choose $q_{0}$ at will and the energy $\left(k_{4}^{0}\right)$ is scalar, we now have two more expressions that are able to give $\Gamma_{\bar{c} c \sigma A}$ and $\Gamma_{\bar{c} c \sigma \sigma}$ in terms of all the other previously considered Green's functions involving the $\sigma$-field except one: $\Gamma_{\bar{c} c \bar{c} c \sigma}$. Also as before, one can immediately verify this identity at tree-level using the Feynman rules of Ref. [28] (and repeated in Appendix A).

\section{Two pairs of further ghost derivatives}

In order to close the system of Slavnov-Taylor identities, we must find an equation for $\Gamma_{\bar{c} c \bar{c} c \sigma}$. This equation arises by functionally differentiating Eq. (2.26) with respect to $\imath c_{w}^{e}, \imath \bar{c}_{v}^{f}, \imath c_{u}^{g}$ and $\imath \bar{c}_{r}^{h}$ and then setting sources to zero. Using the results Eqs. (3.9) and (3.13) and the appropriate functional derivatives of Eq. (2.18) we obtain Eq. (B.4) (the new kernel will be discussed below). This expression is cyclic symmetric in the three ghost derivatives $\imath c_{z}^{d}, \imath c_{w}^{e}$ and $\imath c_{u}^{g}$ and is antisymmetric in the two derivatives $i \bar{c}_{v}^{f}$ and $\imath \bar{c}_{r}^{h}$. In momentum space, the identity reads:

$$
\begin{aligned}
& 0=k_{5}^{0} \Gamma_{\bar{c} c \bar{c} c \sigma}^{h g f d e}\left(k_{1}, k_{2}, k_{3}, k_{4}, k_{5}\right)-\frac{\imath k_{5 i}}{\vec{k}_{5}^{2}} \Gamma_{\bar{c} c \bar{c} c A i}^{h g f d a}\left(k_{1}, k_{2}, k_{3}, k_{4}, k_{5}\right) \Gamma_{\bar{c} c}^{a e}\left(-\vec{k}_{5}\right) \\
& +\left(k_{2}^{0}+q_{0}\right) \Gamma_{\bar{c} c \bar{c} c \sigma}^{h d f e g}\left(k_{1}, k_{4}, k_{3}, k_{5}-q_{0}, k_{2}+q_{0}\right)-\frac{\imath k_{2 i}}{\vec{k}_{2}^{2}} \Gamma_{\bar{c} c \bar{c} c A i}^{h d f e a}\left(k_{1}, k_{4}, k_{3}, k_{5}-q_{0}, k_{2}+q_{0}\right) \Gamma_{\bar{c} c}^{a g}\left(-\vec{k}_{2}\right) \\
& +\left(k_{4}^{0}+q_{0}\right) \Gamma_{\bar{c} c \bar{c} c \sigma}^{h e f g d}\left(k_{1}, k_{5}-q_{0}, k_{3}, k_{2}, k_{4}+q_{0}\right)-\frac{\imath k_{4 i}}{\vec{k}_{4}^{2}} \Gamma_{\bar{c} c \bar{c} c A i}^{h e f g a}\left(k_{1}, k_{5}-q_{0}, k_{3}, k_{2}, k_{4}+q_{0}\right) \Gamma_{\bar{c} c}^{a d}\left(-\vec{k}_{4}\right) \\
& +\Gamma_{\bar{c} c \sigma}^{f d a}\left(k_{3}, k_{4},-k_{3}-k_{4}\right) \tilde{\Gamma}_{\sigma ; \bar{c} c c \bar{c}}^{a e g h}\left(q_{0}+k_{3}+k_{4}, k_{5}-q_{0}, k_{2}, k_{1}\right) \\
& +\Gamma_{\bar{c} c \sigma}^{f e a}\left(k_{3}, k_{5}-q_{0}, q_{0}-k_{3}-k_{5}\right) \tilde{\Gamma}_{\sigma ; \bar{c} c c \bar{c}}^{a g d h}\left(k_{3}+k_{5}, k_{2}, k_{4}, k_{1}\right) \\
& +\Gamma_{\bar{c} c \sigma}^{f g a}\left(k_{3}, k_{2},-k_{2}-k_{3}\right) \tilde{\Gamma}_{\sigma ; \bar{c} c c \bar{c}}^{a d e h}\left(q_{0}+k_{2}+k_{3}, k_{4}, k_{5}-q_{0}, k_{1}\right) \\
& +\Gamma_{\bar{c} c A i}^{f d a}\left(k_{3}, k_{4},-k_{3}-k_{4}\right) \tilde{\Gamma}_{A ; \bar{c} c c \bar{c} i}^{a e g h}\left(q_{0}+k_{3}+k_{4}, k_{5}-q_{0}, k_{2}, k_{1}\right) \\
& +\Gamma_{\bar{c} c A i}^{f e a}\left(k_{3}, k_{5}-q_{0}, q_{0}-k_{3}-k_{5}\right) \tilde{\Gamma}_{A ; \bar{c} c c \bar{c} i}^{a g d h}\left(k_{3}+k_{5}, k_{2}, k_{4}, k_{1}\right) \\
& +\Gamma_{\bar{c} c A i}^{f g a}\left(k_{3}, k_{2},-k_{2}-k_{3}\right) \tilde{\Gamma}_{A ; \bar{c} c c c \bar{c} i}^{a d e h}\left(q_{0}+k_{2}+k_{3}, k_{4}, k_{5}-q_{0}, k_{1}\right) \\
& -\Gamma_{\bar{c} c \sigma}^{h d a}\left(k_{1}, k_{4},-k_{1}-k_{4}\right) \tilde{\Gamma}_{\sigma ; \bar{c} c c \bar{c}}^{a e g f}\left(q_{0}+k_{1}+k_{4}, k_{5}-q_{0}, k_{2}, k_{3}\right) \\
& -\Gamma \bar{c} c c a\left(k_{1}, k_{5}-q_{0}, q_{0}-k_{1}-k_{5}\right) \tilde{\Gamma}_{\sigma ; \bar{c} c c \bar{c}}^{a g d f}\left(k_{1}+k_{5}, k_{2}, k_{4}, k_{3}\right) \\
& -\Gamma_{\bar{c} c \sigma}^{h g a}\left(k_{1}, k_{2},-k_{1}-k_{2}\right) \tilde{\Gamma}_{\sigma ; \bar{c} c c \bar{c}}^{a d e f}\left(q_{0}+k_{1}+k_{2}, k_{4}, k_{5}-q_{0}, k_{3}\right) \\
& -\Gamma_{\bar{c} c A i}^{h d a}\left(k_{1}, k_{4},-k_{1}-k_{4}\right) \tilde{\Gamma}_{A ; \bar{c} c c \bar{c} i}^{a e g f}\left(q_{0}+k_{1}+k_{4}, k_{5}-q_{0}, k_{2}, k_{3}\right) \\
& -\Gamma_{\bar{c} c A i}^{h e a}\left(k_{1}, k_{5}-q_{0}, q_{0}-k_{1}-k_{5}\right) \tilde{\Gamma}_{A ; \bar{c} c c c \bar{c} i}^{a g d f}\left(k_{1}+k_{5}, k_{2}, k_{4}, k_{3}\right) \\
& -\Gamma_{\bar{c} c A i}^{h g a}\left(k_{1}, k_{2},-k_{1}-k_{2}\right) \tilde{\Gamma}_{A ; \bar{c} c c \bar{c} i}^{a d e f}\left(q_{0}+k_{1}+k_{2}, k_{4}, k_{5}-q_{0}, k_{3}\right) \\
& +\frac{1}{2} \Gamma_{\bar{c} c \bar{c} c}^{h d f}\left(k_{1}, k_{4}, k_{3}, k_{2}+k_{5}\right)\left[\tilde{\Gamma}_{\bar{c} ; \bar{c} c c}^{a e g}\left(q_{0}-k_{2}-k_{5}, k_{5}-q_{0}, k_{2}\right)-2 g f^{a e g}\right] \\
& +\frac{1}{2} \Gamma_{\bar{c} c \bar{c} c}^{h e f a}\left(k_{1}, k_{5}-q_{0}, k_{3}, q_{0}+k_{2}+k_{4}\right)\left[\tilde{\Gamma}_{\bar{c} ; \bar{c} c c}^{a g d}\left(-k_{2}-k_{4}, k_{2}, k_{4}\right)-2 g f^{a g d}\right] \\
& +\frac{1}{2} \Gamma_{\bar{c} c \bar{c} c}^{h g f a}\left(k_{1}, k_{2}, k_{3}, k_{4}+k_{5}\right)\left[\tilde{\Gamma}_{\bar{c} ; \bar{c} c c}^{a d e}\left(q_{0}-k_{4}-k_{5}, k_{4}, k_{5}-q_{0}\right)-2 g f^{a d e}\right]
\end{aligned}
$$




$$
\left.+\frac{1}{2} \Gamma_{\bar{c} c}^{f a}\left(\vec{k}_{3}\right) \tilde{\Gamma}_{\bar{c} ; \bar{c} c c c \bar{c}}^{a d q_{0}}+k_{3}, k_{4}, k_{5}-q_{0}, k_{2}, k_{1}\right)-\frac{1}{2} \Gamma_{\bar{c} c}^{h a}\left(\vec{k}_{1}\right) \tilde{\Gamma}_{\bar{c} ; \bar{c} c c c \bar{c}}^{a d e g f}\left(q_{0}+k_{1}, k_{4}, k_{5}-q_{0}, k_{2}, k_{3}\right) .
$$

Aside from the Green's function that we wish to calculate $\left(\Gamma_{\bar{c} c \bar{c} c \sigma}\right)$, there is only one further unknown kernel: $\tilde{\Gamma}_{\bar{c} ; \bar{c} c c c \bar{c}}$, given in Eq. (B.5). Importantly, this kernel introduces no new Green's functions involving functional derivatives with respect to the $\sigma$-field and in momentum space, it reads:

$$
\begin{aligned}
& \tilde{\Gamma}_{\bar{c} ; \bar{c} c c c \bar{c}}^{a d e g f}\left(p_{1}, p_{2}, p_{3}, p_{4}, p_{5}\right)=g f^{a b c} \int d k W_{\bar{c} c}^{b \mu}(k) W_{\bar{c} c}^{c \nu}\left(p_{1}-k\right)\left\{-\frac{1}{3} \Gamma_{\bar{c} c \bar{c} c \bar{c} c}^{\mu g f e d}\left(k, p_{4}, p_{5}, p_{3}, p_{1}-k, p_{2}\right)\right. \\
& +2 \Gamma_{\bar{c} c \bar{c} c}^{f \kappa \nu d}\left(p_{5}, k+p_{3}+p_{4}, p_{1}-k, p_{2}\right) W_{\bar{c} c}^{\kappa \varepsilon}\left(-k-p_{3}-p_{4}\right) \Gamma_{\bar{c} c \bar{c} c}^{\mu g \varepsilon e}\left(k, p_{4},-k-p_{3}-p_{4}, p_{3}\right) \\
& +2 \Gamma_{\bar{c} c \bar{c} c \beta}^{f d \nu g \beta}\left(p_{5}, p_{2}, p_{1}-k, p_{4}, k+p_{3}\right) W_{\alpha \beta}^{\alpha \beta}\left(k+p_{3}\right) \Gamma_{\bar{c} c \alpha}^{\mu e \alpha}\left(k, p_{3},-k-p_{3}\right) \\
& +2 \Gamma_{\bar{c} c \gamma \beta}^{f d \gamma \beta}\left(p_{5}, p_{2}, p_{1}+p_{3}-k, k+p_{4}\right) \Gamma_{\bar{c} c \alpha}^{\mu g \alpha}\left(k, p_{4},-k-p_{4}\right) W_{\alpha \beta}^{\alpha \beta}\left(k+p_{4}\right) W_{\gamma \delta}^{\gamma \delta}\left(k-p_{1}-p_{3}\right) \Gamma_{\bar{c} c \delta}^{\nu e \delta}\left(p_{1}-k, p_{3}, k-p_{1}-p_{3}\right) \\
& +2 \Gamma_{\bar{c} c \beta}^{f \kappa \beta}\left(p_{5},-k-p_{2}-p_{5}, k+p_{2}\right) \Gamma_{\bar{c} c \alpha}^{\mu d \alpha}\left(k, p_{2},-k-p_{2}\right) \Gamma_{\bar{c} c \delta}^{\nu e \delta}\left(p_{1}-k, p_{3}, k-p_{1}-p_{3}\right) \Gamma_{\bar{c} c \gamma}^{\varepsilon g \gamma}\left(k+p_{2}+p_{5}, p_{4}, p_{1}+p_{3}-k\right) \\
& \times W_{\alpha \beta}^{\alpha \beta}\left(k+p_{2}\right) W_{\gamma \delta}^{\gamma \delta}\left(k-p_{1}-p_{3}\right) W_{\bar{c} c}^{\kappa \varepsilon}\left(k+p_{2}+p_{5}\right) \\
& +2 \Gamma_{\bar{c} c \gamma}^{f \kappa \gamma}\left(p_{5}, k+p_{2}+p_{4}, p_{1}+p_{3}-k\right) \Gamma_{\bar{c} c \alpha}^{\mu d \alpha}\left(k, p_{2},-k-p_{2}\right) \Gamma_{\bar{c} c \delta}^{\nu e \delta}\left(p_{1}-k, p_{3}, k-p_{1}-p_{3}\right) \Gamma_{\bar{c} c \beta}^{\varepsilon g \beta}\left(-k-p_{2}-p_{4}, p_{4}, k+p_{2}\right) \\
& \times W_{\alpha \beta}^{\alpha \beta}\left(k+p_{2}\right) W_{\gamma \delta}^{\gamma \delta}\left(k-p_{1}-p_{3}\right) W_{\bar{c} c}^{\kappa \varepsilon}\left(-k-p_{2}-p_{4}\right) \\
& -2 \Gamma_{\bar{c} c \beta}^{f \kappa \beta}\left(p_{5},-k-p_{3}-p_{5}, k+p_{3}\right) \Gamma_{\bar{c} c \bar{c} c}^{\varepsilon d \nu g}\left(k+p_{3}+p_{5}, p_{2}, p_{1}-k, p_{4}\right) \Gamma_{\bar{c} c \alpha}^{\mu e \alpha}\left(k, p_{3},-k-p_{3}\right) \\
& \times W_{\alpha \beta}^{\alpha \beta}\left(k+p_{3}\right) W_{\bar{c} c}^{\kappa \varepsilon}\left(k+p_{3}+p_{5}\right) \\
& +2 \Gamma_{\bar{c} c \bar{c} c}^{f \kappa \nu d}\left(p_{5}, k+p_{3}+p_{4}, p_{1}-k, p_{2}\right) \Gamma_{\bar{c} c \alpha}^{\mu e \alpha}\left(k, p_{3},-k-p_{3}\right) \Gamma_{\bar{c} c \beta}^{\varepsilon g \beta}\left(-k-p_{3}-p_{4}, p_{4}, k+p_{3}\right) \\
& \times W_{\alpha \beta}^{\alpha \beta}\left(k+p_{3}\right) W_{\bar{c} c}^{\kappa \varepsilon}\left(-k-p_{3}-p_{4}\right) \\
& -2 \Gamma_{\bar{c} c \bar{c} c}^{f \kappa \nu d}\left(p_{5}, k+p_{3}+p_{4}, p_{1}-k, p_{2}\right) \Gamma_{\bar{c} c \alpha}^{\mu g \alpha}\left(k, p_{4},-k-p_{4}\right) \Gamma_{\bar{c} c \beta}^{\varepsilon e \beta}\left(-k-p_{3}-p_{4}, p_{3}, k+p_{4}\right) \\
& \left.\times W_{\alpha \beta}^{\alpha \beta}\left(k+p_{4}\right) W_{\bar{c} c}^{\kappa \varepsilon}\left(-k-p_{3}-p_{4}\right)+\text { c.p. }\left(c^{d}\left(p_{2}\right), c^{e}\left(p_{3}\right), c^{g}\left(p_{4}\right)\right)\right\}
\end{aligned}
$$

where we utilize the cyclic symmetry (cyclic symmetric terms denoted by c.p. and this includes the first factor $\Gamma_{\bar{c} c \bar{c} c \bar{c} c}$ which is the origin of the factor 1/3). Because of the cyclic symmetry and the fact that $q_{0}$ is arbitrary, Eq. (4.17) can be solved for $\Gamma_{\bar{c} c \bar{c} c \sigma}$ and we have finally managed to close the set of Slavnov-Taylor identities. The identity, Eq. (4.17), trivially has no tree-level form.

We have thus shown that just as for the temporal two-point functions, all temporal Green's functions considered so far can be deduced (at least in principle) from their non-temporal counterparts as solutions to the Slavnov-Taylor identities. The temporal $\sigma$-field has been effectively eliminated from the system, or integrated out of functional form of the action. Again, as for the two-point functions we see that the Slavnov-Taylor identities relate temporal, (spatially) longitudinal and ghost Green's functions in a manner reminiscent of the Kugo-Ojima quartet mechanism [18].

\section{Further Slavnov-Taylor identities}

It has been shown so far that there exists a closed set of Slavnov-Taylor identities that includes the vertex (threepoint proper) Green's functions. However, one may also consider four-point functions (e.g., $\left.\Gamma_{A A A \sigma}\right)$. Because of their extended nature, we do not attempt to derive these identities completely; rather, we shall merely sketch their form in order to justify that the equations close just as before. We begin as previously with Eq. (2.26) and again, the sets of derivatives are distinguished by how many pairs of ghost/antighost functional derivatives are taken. We will highlight only those temporal Green's functions or kernels that have not previously appeared and leave those quantities that have already been derived as implicit. The sequence is as follows:

1. Taking functional derivatives of Eq. (2.26) with respect to $\imath \Phi_{\kappa}, \imath \Phi_{\tau}$ and $\imath \Phi_{\lambda}$ (as before, the ghost derivatives will be made explicit and the field types here refer only to either the $\vec{A}$ or $\sigma$-fields), one clearly obtains an identity for $\Gamma_{\kappa \tau \lambda \sigma}$ in terms of $\Gamma_{\kappa \tau \lambda A}$ and a new kernel $\tilde{\Gamma}_{\sigma ; \bar{c} c \lambda \tau}$. This kernel, following (as a further functional derivative of) Eq. (4.7) introduces the new quantity: $\Gamma_{\bar{c} c \kappa \tau \lambda}$. Starting with $\Gamma_{A A A A}$, we can then sequentially build up to $\Gamma_{\sigma \sigma \sigma \sigma}$, given all the $\Gamma_{\bar{c} c \kappa \tau \lambda}$.

2. Next we take one pair of ghost functional derivatives (i.e., functionally differentiate Eq. (2.26) with respect to $\imath \bar{c}$ and $\imath c)$ and then derivatives with respect to $\imath \Phi_{\tau}$ and $\imath \Phi_{\lambda}$. This gives us equations for the $\Gamma_{\bar{c} c \tau \lambda \sigma}$ in terms of 
the $\Gamma_{\bar{c} c \tau \lambda A}$ and we have two new kernels: $\tilde{\Gamma}_{\sigma ; \bar{c} c c \bar{c} \lambda}$ and $\tilde{\Gamma}_{\bar{c} ; \bar{c} c c \lambda \tau}$. These kernels follow from Eqs. (B.2) and (B.3), respectively, and both introduce the new function: $\Gamma_{\bar{c} c \bar{c} c \tau \lambda}$.

3. With two pairs of ghost functional derivatives of Eq. (2.26), plus one further derivative with respect to $\imath \Phi_{\lambda}$ we

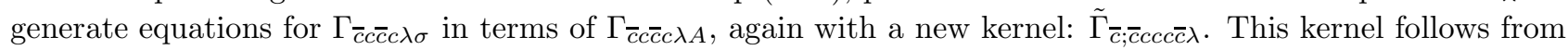

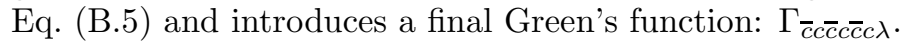

4. We finally take three pairs of ghost functional derivatives to get an equation for $\Gamma_{\bar{c} c \bar{c} c \bar{c} c \sigma}$ in terms of $\Gamma_{\bar{c} c \bar{c} c \bar{c} c A}$. However, this equation does not involve any further kernels and the set of equations terminates.

Now, all these equations have the same characteristics as in the previous subsections: the energy is a scalar quantity and there is the energy injection scale $q_{0}$, such that one has a set of unambiguous (albeit nonlinear and extremely long) equations from which the temporal Green's functions may be derived, given the set of spatial and ghost Green's functions as external input.

We conjecture that in principle, the closed sets of Slavnov-Taylor identities in Coulomb gauge may be extended to include all higher $n$-point Green's functions and that they may be solved to give exact expressions for all the temporal Green's functions. As may be appreciated though, how to prove such a general statement is not clear. One may formulate some ideas based on the following observations: Firstly, the scalar nature of the energy and the energy injection scale $\left(q_{0}\right)$ will always be present in the Slavnov-Taylor identities of Coulomb gauge courtesy of the particular Gauss-BRST invariance of the theory (this certainly improves on the situation in linear covariant gauges where one has contractions of tensors such that transverse parts cannot be directly deduced). Second, because ghost derivatives must come in pairs, each further pair reduces the number of possible non-ghost internal lines within the kernels - the external ghost legs must be connected by an internal ghost propagator or a vertex with these two ghost legs. This restricts the number of possible internal temporal Green's functions such that eventually the set of identities closes. One can see the emergence of a characteristic pattern for the ghost functions necessary to form the kernels in the steps above: i.e., (1.) $\Gamma_{\bar{c} c \kappa \tau \lambda} \rightarrow(2.) \Gamma_{\bar{c} c \bar{c} c \tau \lambda} \rightarrow(3.) \Gamma_{\bar{c} c \bar{c} c \bar{c} c \lambda} \rightarrow$ (4.) 0 .

\section{SIMPLIFICATIONS OF THE IDENTITIES}

Given that the Slavnov-Taylor identities derived in previous sections relate the various types of Green's functions in an extended manner, it is pertinent to ask whether these relationships reduce in specific circumstances such that unambiguous statements can be made about the behavior of individual Green's functions, in particular the two-point functions. The motivation is clear: such information (particularly in the infrared region) may be useful for determining how the confinement mechanism manifests itself (asymptotic freedom already being perturbatively established in the ultraviolet region of Coulomb gauge [28]) and also provides for input in other nonperturbative studies. We argue here that unfortunately such information cannot be unambiguously extracted.

In the noncovariant Coulomb gauge, we must first sort out the temporal (energy) and spatial (momentum) scales. The most obvious simplification of the Slavnov-Taylor identities concerns purely spacelike momenta where we know that if the postulate of Euclidicity is to hold (i.e., that the Wick rotation is valid), the Green's functions presumably can have no singularities. One may in principle also consider timelike configurations, but this is certainly beyond the scope of the present analysis. Thus, we must first set all energy scales to zero. We define the infrared region as the limit as one or more of the momenta vanishes and we approach the light-cone, where singularities (or nontrivial zeroes) may appear.

Let us begin by considering the Slavnov-Taylor identities for the two-point functions: Eqs. (3.16) and (3.17). Using the general decompositions of Ref. [28] (presented also in Appendix A) in terms of the (scalar) dressing functions, we get for general kinematical configurations:

$$
\begin{aligned}
-\imath k_{0} \vec{k}^{2} \Gamma_{\sigma \sigma}\left(k_{0}^{2}, \vec{k}^{2}\right) & =-\imath k_{0} \vec{k}^{2} \Gamma_{A \sigma}\left(k_{0}^{2}, \vec{k}^{2}\right) \Gamma_{\bar{c} c}\left(\vec{k}^{2}\right), \\
\imath k_{0}^{2} k_{k} \Gamma_{A \sigma}\left(k_{0}^{2}, \vec{k}^{2}\right) & =\imath k_{0}^{2} k_{k} \bar{\Gamma}_{A A}\left(k_{0}^{2}, \vec{k}^{2}\right) \Gamma_{\bar{c} c}\left(\vec{k}^{2}\right) .
\end{aligned}
$$

Since both equations have common kinematical prefactors, we can then write

$$
\Gamma_{\sigma \sigma}\left(k_{0}^{2}, \vec{k}^{2}\right)=\Gamma_{A \sigma}\left(k_{0}^{2}, \vec{k}^{2}\right) \Gamma_{\bar{c} c}\left(\vec{k}^{2}\right)=\bar{\Gamma}_{A A}\left(k_{0}^{2}, \vec{k}^{2}\right)\left[\Gamma_{\bar{c} c}\left(\vec{k}^{2}\right)\right]^{2}
$$

This holds for any kinematical configuration and we conclude that the Slavnov-Taylor identities for the two-point functions alone can give no information about the value of the Green's functions, only the relationship between them. 
Slightly less trivial is the Slavnov-Taylor identity for the ghost-gluon vertex, Eq. (4.12). Concentrating on spacelike momenta as discussed above, we see that neither of the $\Gamma_{\bar{c} c \sigma}$ vertices will contribute when the energy is set to zero. To study the equation, let us firstly decompose the ghost-gluon vertex into tree-level and dressed parts as follows:

$$
\Gamma_{\bar{c} c A i}^{a b c}\left(p_{1}, p_{2}, p_{3}\right)=-\imath g f^{a b c} p_{1 i}-\imath g f^{a b c} p_{1 j} \tilde{\Gamma}_{A ; \bar{c} c A j i}\left(p_{1}, p_{2}, p_{3}\right) .
$$

Let us explain this decomposition. Since we are dealing with a three-point function, the color factor $\left(f^{a b c}\right)$ and coupling $(g)$ will always be common and can be extracted. The appearance of $\tilde{\Gamma}$ stems from the momentum space form of Eq. (4.3) and in fact, given the definition Eq. (4.8), the above equation, Eq. (5.3), is the Dyson-Schwinger equation for the spatial ghost-gluon vertex. As will be seen shortly, the contraction of $p_{1 j}$ with $\tilde{\Gamma}_{A ; \bar{c} c A j i}$ is important. Lastly, for the $\tilde{\Gamma}_{\bar{c} ; \bar{c} c c}$ kernel, we can only extract the color and coupling factors and we write

$$
\tilde{\Gamma}_{\bar{c} ; \bar{c} c c}^{a b c}=g f^{a b c} \tilde{\Gamma}_{\bar{c} ; \bar{c} c c}
$$

At zero energy, Eq. (4.12) in terms of dressing functions is thus

$$
\begin{aligned}
0= & -\vec{k}_{1} \cdot \vec{k}_{3} \Gamma_{\bar{c} c}\left(\vec{k}_{3}^{2}\right)-\vec{k}_{1} \cdot \vec{k}_{2} \Gamma_{\bar{c} c}\left(\vec{k}_{2}^{2}\right)-\vec{k}_{1}^{2} \Gamma_{\bar{c} c}\left(\vec{k}_{1}^{2}\right)-k_{1 j} k_{3 i} \tilde{\Gamma}_{A ; \bar{c} c A j i}\left(\vec{k}_{1}, \vec{k}_{2}, \vec{k}_{3}\right) \Gamma_{\bar{c} c}\left(\vec{k}_{3}^{2}\right)-k_{1 j} k_{2 i} \tilde{\Gamma}_{A ; \bar{c} c A j i}\left(\vec{k}_{1}, \vec{k}_{3}, \vec{k}_{2}\right) \Gamma_{\bar{c} c}\left(\vec{k}_{2}^{2}\right) \\
& +\frac{1}{2} \vec{k}_{1}^{2} \Gamma_{\bar{c} c}\left(\vec{k}_{1}^{2}\right) \tilde{\Gamma}_{\bar{c} ; \bar{c} c c}\left(\vec{k}_{1}, \vec{k}_{2}, \vec{k}_{3}\right) .
\end{aligned}
$$

This equation is directly analogous to the Landau gauge Slavnov-Taylor identity for the ghost-gluon vertex which was studied under the truncation $\tilde{\Gamma}_{\bar{c} ; \bar{c} c c}=0$ [12]. Quite generally, it states that the kernels $\left(\tilde{\Gamma}_{A ; \bar{c} c A j i}\right.$ and $\left.\tilde{\Gamma}_{\bar{c} ; \bar{c} c c}\right)$ and the two-point ghost dressing function $\Gamma_{\bar{c} c}$ are nontrivially related.

Let us now discuss the above identity, Eq. (5.5), in the infrared to assess whether it simplifies further. Because of the symmetry, there are two limits of interest: $k_{1} \rightarrow 0$ and $k_{2} \rightarrow 0$. For the infrared limit $k_{1} \rightarrow 0$, we see that the entire equation has an overall factor $\sim\left|k_{1}\right|$ (in fact, one can show that there is the overall factor $\left|k_{1}\right|^{2}$ ). Therefore, in the limit, no single Green's function is isolated from which to determine a value for this kinematical configuration. Further, the Green's functions or their combination may even be singular. For the limit $k_{2} \rightarrow 0$, we recall that in Coulomb gauge, ghost vertex dressing functions vanish as the "in-ghost" momentum vanishes [27], i.e.,

$$
\begin{array}{rll}
\tilde{\Gamma}_{A ; \bar{c} c A j i}\left(k_{1}, k_{2}, k_{3}\right) & \stackrel{\vec{k}_{2} \rightarrow 0}{\propto} & \left|\vec{k}_{2}\right|, \\
\tilde{\Gamma}_{\bar{c} ; \bar{c} c c}\left(k_{1}, k_{2}, k_{3}\right) & \stackrel{\vec{k}_{2} \rightarrow 0}{\propto} & \left|\vec{k}_{2}\right| .
\end{array}
$$

This is, of course, exactly the same as in Landau gauge [2]. Using the momentum conservation, $k_{3}=-k_{1}-k_{2}$, and we see that Eq. (5.5) has the overall factor $\left|k_{2}\right|$ as $k_{2} \rightarrow 0$. This leads to the conclusion that no information about an individual Green's function may be extracted in this limit either. We conclude that on its own, the Slavnov-Taylor identity for the ghost-gluon vertex, Eq. (4.12), does not yield unambiguous information about the Green's functions without further knowledge.

For the Slavnov-Taylor identities given by Eq. (4.6), there is one special case where simplification occurs and this again results in a situation exactly analogous to Landau gauge. Considering the $\Gamma_{A A \sigma}$ identity (i.e., setting the external indices $\lambda$ and $\tau$ to be referring to $\vec{A}$-fields), one has explicitly

$$
\begin{aligned}
k_{3}^{0} \Gamma_{A A \sigma l k}^{f e d}\left(k_{1}, k_{2}, k_{3}\right)= & \imath \frac{k_{3 i}}{\vec{k}_{3}^{2}} \Gamma_{3 A l k i}^{f e a}\left(k_{1}, k_{2}, k_{3}\right) \Gamma_{\bar{c} c}^{a d}\left(-\vec{k}_{3}\right) \\
& -\Gamma_{A A k i}^{e a}\left(k_{2}\right)\left[\tilde{\Gamma}_{A ; \bar{c} c A i l}^{a d f}\left(k_{2}+q_{0}, k_{3}-q_{0}, k_{1}\right)+g f^{a d f} \delta_{i l}\right] \\
& -\Gamma_{A A l i}^{f a}\left(k_{1}\right)\left[\tilde{\Gamma}_{A ; \bar{c} c A i k}^{a d e}\left(k_{1}+q_{0}, k_{3}-q_{0}, k_{2}\right)+g f^{a d e} \delta_{i k}\right] \\
& -\Gamma_{A \sigma k}^{e a}\left(k_{2}\right) \tilde{\Gamma}_{\sigma ; \bar{c} c A l}^{a d f}\left(k_{2}+q_{0}, k_{3}-q_{0}, k_{1}\right) \\
& -\Gamma_{A \sigma l}^{f a}\left(k_{1}\right) \tilde{\Gamma}_{\sigma ; \bar{c} c A k}^{a d e}\left(k_{1}+q_{0}, k_{3}-q_{0}, k_{2}\right) .
\end{aligned}
$$

Further setting all energy scales to zero and using the general decompositions $\left(\Gamma_{3 A}^{a b c}=-\imath g f^{a b c} \Gamma_{3 A}\right.$, for the rest, see above or Appendix A) one has

$$
\begin{aligned}
k_{3 i} \Gamma_{3 A l k i}\left(\vec{k}_{1}, \vec{k}_{2}, \vec{k}_{3}\right) \Gamma_{\bar{c} c}\left(\vec{k}_{3}^{2}\right)= & \vec{k}_{2}^{2} t_{k l}\left(\vec{k}_{2}\right) \Gamma_{A A}\left(\vec{k}_{2}^{2}\right)+\vec{k}_{2}^{2} t_{k i}\left(\vec{k}_{2}\right) \Gamma_{A A}\left(\vec{k}_{2}^{2}\right) \tilde{\Gamma}_{A ; \bar{c} c A i l}\left(\vec{k}_{2}, \vec{k}_{3}, \vec{k}_{1}\right) \\
& -\vec{k}_{1}^{2} t_{l k}\left(\vec{k}_{1}\right) \Gamma_{A A}\left(\vec{k}_{1}^{2}\right)-\vec{k}_{1}^{2} t_{l i}\left(\vec{k}_{1}\right) \Gamma_{A A}\left(\vec{k}_{1}^{2}\right) \tilde{\Gamma}_{A ; \bar{c} c A i k}\left(\vec{k}_{1}, \vec{k}_{3}, \vec{k}_{2}\right) .
\end{aligned}
$$


Taking the contraction of the above equation with $k_{2 k}$, one has thus

$$
k_{2 k} k_{3 i} \Gamma_{3 A l k i}\left(\vec{k}_{1}, \vec{k}_{2}, \vec{k}_{3}\right)=-\vec{k}_{1}^{2} \frac{\Gamma_{A A}\left(\vec{k}_{1}^{2}\right)}{\Gamma_{\bar{c} c}\left(\vec{k}_{3}^{2}\right)} t_{l j}\left(\vec{k}_{1}\right)\left[k_{2 j}+k_{2 k} \tilde{\Gamma}_{A ; \bar{c} c A j k}\left(\vec{k}_{1}, \vec{k}_{3}, \vec{k}_{2}\right)\right]
$$

Now, the overall Bose-symmetry of the three-gluon vertex means that after extracting the color factor $\left(f^{a b c}\right)$, the dressing function $\Gamma_{3 A}$ is antisymmetric under exchange of any two legs and so, by interchanging $k_{2 k} \leftrightarrow k_{3 i}$ one can eliminate the three-gluon vertex to obtain an expression involving only the two-point ghost dressing function $\left(\Gamma_{\bar{c}}\right)$ and the kernel $\tilde{\Gamma}_{A ; \bar{c} c A j k}$ which, after canceling out the overall factors reads:

$$
0=t_{l j}\left(\vec{k}_{1}\right)\left\{k_{2 j} \Gamma_{\bar{c} c}\left(\vec{k}_{2}^{2}\right)+k_{3 j} \Gamma_{\bar{c} c}\left(\vec{k}_{3}^{2}\right)+\Gamma_{\bar{c} c}\left(\vec{k}_{2}^{2}\right) k_{2 k} \tilde{\Gamma}_{A ; \bar{c} c A j k}\left(\vec{k}_{1}, \vec{k}_{3}, \vec{k}_{2}\right)+\Gamma_{\bar{c} c}\left(\vec{k}_{3}^{2}\right) k_{3 k} \tilde{\Gamma}_{A ; \bar{c} c A j k}\left(\vec{k}_{1}, \vec{k}_{2}, \vec{k}_{3}\right)\right\}
$$

In Landau gauge, this identity is well-known [35, 36] and was considered in Ref. [22] under reasonable assumptions to be indicative of an infrared finite ghost dressing function $\left(\Gamma_{\bar{c} c}\right)$. In the infrared limit $k_{2} \rightarrow 0$ (equivalently for $k_{3} \rightarrow 0$ ), we see that all terms have the prefactor $\left|k_{2}\right|$ since $k_{3 j} t_{l j}\left(\vec{k}_{1}\right)=-k_{2 j} t_{l j}\left(\vec{k}_{1}\right)$ and for the kernel we have the general infrared result Eq. (5.6). Without further assumption, the following (conservative) statement is true of Eq. (5.10): if the particular contraction above of the ghost-gluon vertex kernel $\left(\tilde{\Gamma}_{A ; \bar{c} c A j k}\right)$ is vanishing, then the ghost dressing function is constant. However, notice that the 'particular contraction' above is not the same as that appearing in either the Slavnov-Taylor identity for the ghost-gluon vertex, Eq. (5.5) or the Dyson-Schwinger equations from which $\Gamma_{\bar{c} c}$ can be obtained. Thus we conclude, as previously, that the above component, Eq. (5.10), of the Slavnov-Taylor identity, Eq. (4.6) does not yield unambiguous information about particular Green's functions without further input.

The special symmetric contraction case (above) of the three-gluon vertex Slavnov-Taylor identity aside, in order to say anything about the two-point functions from the identities Eq. (4.6), one must know something about at least one of the vertices $\Gamma_{\tau \lambda \sigma}$ or $\Gamma_{\tau \lambda A}$. Such information is not available from general considerations and so, one cannot make any simple statement. Further, the rest of the Slavnov-Taylor identities, equations (4.14) and (4.17), clearly involve too many higher $n$-point functions to have any hope of simplification.

From the above discussion, it seems clear that no information about the behavior of the two-point functions (or vertices) can be obtained from the Slavnov-Taylor identities alone. As is obvious from the simple Slavnov-Taylor identities for the two-point functions summarized by Eq. (5.2), one can only determine the relationship between the various Green's functions and this must be true for any kinematical configuration. Our only unambiguous 'outside' knowledge about the Green's functions was the peculiar infrared behavior of the ghost vertices, the absence of singularities for spacelike momenta and the Bose-symmetry of the three-gluon vertex (which is why the ghost-gluon vertex identity, Eq. (4.12), and the identity Eq. (5.10) were of particular interest), but even then, the dimensionality or complexity of the Slavnov-Taylor identities denied concrete conclusions.

\section{SUMMARY, DISCUSSION AND CONCLUSIONS}

In this paper, the Slavnov-Taylor identities for Coulomb gauge Yang-Mills theory within the second order formalism have been derived. The starting point was the Gauss-BRST invariance of the action [21], characterized by the time-dependent BRS variation and peculiar to Coulomb gauge. It was found that for the two-point and vertex Green's functions, the Slavnov-Taylor identities form closed sets from which the temporal Green's functions can be unambiguously derived given the relevant spatial gluon and ghost Green's functions as input. The extension of this to higher order Green's functions was also discussed. Special cases of the identities were studied and it was seen that there is no simplification such that information about a particular Green's function could be extracted.

It is worthwhile at this stage to discuss the Coulomb gauge Slavnov-Taylor identities in their wider context since the connection between several different themes becomes apparent. The first of these themes centers around Gauss' law. In classical electrodynamics, Gauss' law allows one to determine the temporal component of the gauge field (i.e., $\sigma)$ directly from the physical charge distribution. In the canonical (Hamiltonian-based) formulation of Yang-Mills theory, Gauss' law is applied as an operator identity to define the physical state space and can be explicitly resolved in Coulomb gauge, yielding the so-called Coulomb term which comprises the confining properties of the theory [25]. In the functional formalism, Gauss' law appears after the elimination of the temporal $(\sigma)$ field from the action, either directly as in the case of the first order formalism [21, 32] or as here as the elimination from the effective action via the Slavnov-Taylor identities. The resolution of Gauss' law and its connection to the physical state space is certainly less apparent in the functional formalism since Green's functions are not directly related to physical observables but this is compensated for by the observation that the cancellation of the gauge-dependent degrees of freedom (i.e., temporal, longitudinal and ghost) is manifest — in the first order formalism one can reduce the functional integral 
to transverse spatial gluon degrees of freedom and the Slavnov-Taylor identities here express this explicitly for the Green's functions in local fashion. However, the connection between the physical state space and the Green's functions is understood conceptually within the framework of the Kugo-Ojima confinement scenario [18]: by postulating a welldefined BRS charge and physical state space, the cancellation of the gauge-dependent degrees of freedom followed and in Landau gauge led to the prediction that the ghost propagator is infrared enhanced. In the Coulomb gauge functional formalism, we know explicitly that there exists at least a total charge that is conserved and vanishing [32] which partly confirms the Kugo-Ojima postulate. This total charge arises from considering the temporal zeromodes inherent to Coulomb gauge and leads to the formal demonstration of the cancellation of the gauge dependent degrees of freedom. In this study, we have shown that the Slavnov-Taylor identities stemming from the temporally nontrivial Gauss-BRST transform supply this cancellation in local fashion. In this respect, the temporal features of the Coulomb gauge functional formalism can be seen to supply a link between the physical charge and states to the Green's functions of the underlying theory.

The temporally nontrivial nature of Coulomb gauge is manifested in two ways. On the one hand, resolving the temporal zero-modes leads (as mentioned above) to the vanishing and conserved total charge [32]; on the other hand, the extra temporal degree of freedom in the Gauss-BRST transform leads to the closure of the Slavnov-Taylor identities. The temporal zero-modes of the Faddeev-Popov operator lead us to consider the connection to the GribovZwanziger picture of confinement [19-21]. In this scenario, it is recognized that the zero-modes (which are induced by incomplete gauge-fixing) should be separated from the functional integration and via stochastic quantization and entropy arguments, the authors were able to show that the resulting spatial transverse gluon propagator would be suppressed in the infrared (and thus drops out from the physical spectrum) whereas the temporal propagator provided for a long-range confining force. Just as with the Kugo-Ojima scenario, the ghost propagator in Landau gauge would also be infrared enhanced. In the Coulomb gauge functional formalism insofar as the temporal zero-modes are concerned, one can see the parallels: their resolution leads to a total charge and the cancellation of gauge degrees of freedom, providing an explicit demonstration of selected features of both the Gribov-Zwanziger and Kugo-Ojima confinement scenarios. However, one part is evidently missing - a prediction for the infrared behavior of the ghost (or something similar). Resolving the temporal zero-modes in Coulomb gauge restricted the functional integral to field configurations such that the total charge is conserved and vanishing, whereas the same temporal feature gave rise to Slavnov-Taylor identities that form closed sets but explicitly no information about a particular Green's function. This underlies the quite general feature of functional techniques, namely, that in order to talk about Green's functions one must take functional derivatives and in this respect, the Slavnov-Taylor identities (and for that matter, the Dyson-Schwinger equations too) represent functional differential equations. Their solution is known only up to some 'constant' of integration (for explicit examples of this, see Refs. [23, 24, 37] and references therein). In general this is not obvious since one necessarily has an infinite tower of equations to consider, but in the case of the Coulomb gauge Slavnov-Taylor identities derived here, the closure allows us to see clearly that this is precisely the case - functional techniques lead to relations between Green's functions.

Pragmatically, there are two natural avenues which to explore further. The first is to search for the 'missing' prediction about the value of a specific Green's function (most likely the infrared behavior of the ghost) in order to complete the connection between the temporal aspects of Coulomb gauge and the two confinement scenarios: KugoOjima and Gribov-Zwanziger. The second direction to take is to use the Slavnov-Taylor identities here in order to construct a charge-conserving truncation scheme from which to solve the Dyson-Schwinger equations of Coulomb gauge Yang-Mills theory, allowing for the eventuality that the solution may only be determined up to some external 'boundary condition' in analogy to the solution of standard differential equations. Both these directions are being pursued.

On a final note, one further consideration for the Slavnov-Taylor identities derived in this paper is their verification to one-loop order in perturbation theory (the tree-level forms are trivial). This has in fact been done. However, as can be appreciated from the lengthy expressions, such a technical exercise is not suitable for inclusion in the present paper. The verification of the one-loop identities involves three components: the one-loop expansion of the vertex Dyson-Schwinger equations (and the kernels presented in the text), the use of the inherent translational invariance of the loop integrals and identities for the color factors. Importantly, none of the loop integrals need be explicitly evaluated.

\section{Acknowledgments}

This work has been supported by the Deutsche Forschungsgemeinschaft (DFG) under contracts no. DFG-Re856/62,3 . 


\begin{tabular}{|c|c|c|}
\hline$W$ & $A_{j}$ & $\sigma$ \\
\hline$A_{i}$ & $t_{i j}(\vec{k}) \frac{\imath}{\left(k_{0}^{2}-\vec{k}^{2}\right)} D_{A A}$ & 0 \\
\hline$\sigma$ & 0 & $\frac{2}{\vec{k}^{2}} D_{\sigma \sigma}$ \\
\hline$\lambda$ & $\frac{k_{j}}{\vec{k}^{2}}$ & $\frac{k^{0}}{\vec{k}^{2}} D_{\sigma \lambda}$ \\
\hline
\end{tabular}

\begin{tabular}{|c|c|c|}
\hline$\Gamma$ & $A_{j}$ & $\sigma$ \\
\hline$A_{i}$ & $-\imath\left(k_{0}^{2}-\vec{k}^{2}\right) t_{i j}(\vec{k}) \Gamma_{A A}-\imath k_{0}^{2} \frac{k_{i} k_{j}}{\vec{k}^{2}} \bar{\Gamma}_{A A}$ & $\imath k^{0} k_{i} \Gamma_{A \sigma}$ \\
\hline$\sigma$ & $\imath k^{0} k_{j} \Gamma_{A \sigma}$ & $-\imath \vec{k}^{2} \Gamma_{\sigma \sigma}$ \\
\hline$\lambda$ & $-k_{j}$ & 0 \\
\hline
\end{tabular}

TABLE I: General form of propagators [left] and two-point proper functions [right] (without color factors) in momentum space. All dressing functions are functions of $k_{0}^{2}$ and $\vec{k}^{2}$.

\section{APPENDIX A: FEYNMAN RULES AND DECOMPOSITIONS}

For completeness, we present here selected results from Ref. [28] concerning the tree-level forms and general decompositions of various Green's functions. The general decompositions of the non-ghost propagators and proper two-point functions are given in Table I (without the common color factor $\delta^{a b}$ ). For the ghosts, we have

$$
W_{\bar{c} c}^{a b}(k)=-\delta^{a b} \frac{\imath}{\vec{k}^{2}} D_{\bar{c} c}\left(\vec{k}^{2}\right), \quad \Gamma_{\bar{c} c}^{a b}(k)=\delta^{a b} \imath \vec{k}^{2} \Gamma_{\bar{c} c}\left(\vec{k}^{2}\right) .
$$

At tree-level, all dressing functions are unity. The tree-level vertices are given by (all momenta are defined as incoming)

$$
\begin{aligned}
\Gamma_{\sigma A A j k}^{(0) a b c}\left(p_{a}, p_{b}, p_{c}\right) & =\imath g f^{a b c} \delta_{j k}\left(p_{b}^{0}-p_{c}^{0}\right), \\
\Gamma_{\sigma A \sigma j}^{(0) a b c}\left(p_{a}, p_{b}, p_{c}\right) & =-\imath g f^{a b c}\left(p_{a}-p_{c}\right)_{j}, \\
\Gamma_{3 A i j k}^{(0) a b c}\left(p_{a}, p_{b}, p_{c}\right) & =-\imath g f^{a b c}\left[\delta_{i j}\left(p_{a}-p_{b}\right)_{k}+\delta_{j k}\left(p_{b}-p_{c}\right)_{i}+\delta_{k i}\left(p_{c}-p_{a}\right)_{j}\right] \\
\Gamma_{\bar{c} c A i}^{(0) a b c}\left(p_{\bar{c}}, p_{c}, p_{A}\right) & =-\imath g f^{a b c} p_{\bar{c} i} .
\end{aligned}
$$

\section{APPENDIX B: LIST OF LENGTHY FORMULA}

In this appendix, we list lengthy (configuration space) formula, necessary for the derivation of the Slavnov-Taylor identities, that occur in the text.

$$
\begin{aligned}
& 0=\int d^{4} x \delta\left(t-x_{0}\right)\left\{\imath\left[\partial_{x}^{0}<\imath \bar{c}_{v}^{f} \imath c_{z}^{d} \imath \Phi_{\lambda(k) u}^{g} \imath \sigma_{x}^{e}>\right] \delta(w-x)-\imath\left[\partial_{x}^{0}<\imath \bar{c}_{v}^{f} \imath c_{w}^{e} \imath \Phi_{\lambda(k) u}^{g} \imath \sigma_{x}^{d}>\right] \delta(z-x)\right. \\
& -\left[\frac{\nabla_{i x}}{\left(-\nabla_{x}^{2}\right)}<\imath \bar{c}_{v}^{f} \imath c_{z}^{d} \imath \Phi_{\lambda(k) u}^{g} \imath A_{i x}^{a}>\right]<\imath \bar{c}_{x}^{a} \imath c_{w}^{e}>+\left[\frac{\nabla_{i x}}{\left(-\nabla_{x}^{2}\right)}<\imath \bar{c}_{v}^{f} \imath c_{w}^{e} \imath \Phi_{\lambda(k) u}^{g} \imath A_{i x}^{a}>\right]<\imath \bar{c}_{x}^{a} \imath c_{z}^{d}> \\
& +<\imath \bar{c}_{v}^{f} \imath c_{z}^{d} z \sigma_{x}^{a}>\left[\tilde{\Gamma}_{\sigma ; \bar{c} c \lambda(k)}^{a e g}(x, w, u)-g f^{a g e} \delta_{\sigma \lambda} \delta(u-x) \delta(w-x)\right] \\
& -<\imath \bar{c}_{v}^{f} \imath c_{w}^{e} \imath \sigma_{x}^{a}>\left[\tilde{\Gamma}_{\sigma ; \bar{c} c \lambda(k)}^{a d g}(x, z, u)-g f^{a g d} \delta_{\sigma \lambda} \delta(u-x) \delta(z-x)\right] \\
& +<\bar{c} \bar{c}_{v}^{f} \imath c_{z}^{d} \imath A_{i x}^{a}>\left[\tilde{\Gamma}_{A ; \bar{c} c \lambda i(k)}^{a e g}(x, w, u)-g f^{a g e} \delta_{k i} \delta_{A \lambda} \delta(u-x) \delta(w-x)\right] \\
& -<\imath \bar{c}_{v}^{f} \imath c_{w}^{e} \imath A_{i x}^{a}>\left[\tilde{\Gamma}_{A ; \bar{c} c \lambda i(k)}^{a d g}(x, z, u)-g f^{a g d} \delta_{k i} \delta_{A \lambda} \delta(u-x) \delta(z-x)\right] \\
& +\frac{1}{2}<\imath \bar{c}_{v}^{f} \imath c_{x}^{a} \imath \Phi_{\lambda(k) u}^{g}>\left[\tilde{\Gamma}_{\bar{c} ; \bar{c} c c}^{a d e}(x, z, w)-2 g f^{a d e} \delta(z-x) \delta(w-x)\right] \\
& \left.-<\imath \Phi_{\lambda(k) u}^{g} \imath \sigma_{x}^{a}>\tilde{\Gamma}_{\sigma ; \bar{c} c c \bar{c}}^{a d e f}(x, z, w, v)-<\imath \Phi_{\lambda(k) u}^{g} \imath A_{i x}^{a}>\tilde{\Gamma}_{A ; \bar{c} c c \bar{c} i}^{a d e f}(x, z, w, v)+\frac{1}{2}<\imath \bar{c}_{v}^{f} \imath c_{x}^{a}>\tilde{\Gamma}_{\bar{c} ; \bar{c} c c \lambda(k)}^{a d e g}(x, z, w, u)\right\} . \\
& \tilde{\Gamma}_{\sigma ; \bar{c} c c \bar{c}}^{a d e f}(x, z, w, v)=g f^{a b c} \frac{\delta^{3}}{\delta \imath \bar{c}_{v}^{f} \delta \imath c_{w}^{e} \delta \imath c_{z}^{d}}<\imath \rho_{x}^{b} \imath \bar{\eta}_{x}^{c}>\left.\right|_{J=0} \\
& =g f^{a b c}<\imath \rho_{x}^{b} \imath J_{\nu}><\imath \bar{\eta}_{x}^{c} \imath \eta_{\gamma}>\times
\end{aligned}
$$




$$
\begin{aligned}
& \left\{l \bar{c}_{v}^{f} \imath c_{\mu} \imath \Phi_{\nu}><\imath \bar{\eta}_{\mu} \imath \eta_{\varepsilon}><\imath J_{\alpha} \imath J_{\kappa}>\left[<\imath \bar{c}_{\varepsilon} \imath c_{z}^{d} \imath \Phi_{\alpha}><\imath \bar{c}_{\gamma} \imath c_{w}^{e} \imath \Phi_{\kappa}>-<\imath \bar{c}_{\varepsilon} \imath c_{w}^{e} \imath \Phi_{\alpha}><\imath \bar{c}_{\gamma} \imath c_{z}^{d} \imath \Phi_{\kappa}>\right]\right. \\
& +<\imath \bar{c}_{v}^{f} \imath c_{\mu} \imath \Phi_{\varepsilon}><\imath \bar{\eta}_{\mu} \imath \eta_{\alpha}><\imath J_{\varepsilon} \imath J_{\kappa}>\left[<\imath \bar{c}_{\alpha} \imath c_{z}^{d} \imath \Phi_{\nu}><\imath \bar{c}_{\gamma} \imath c_{w}^{e} \imath \Phi_{\kappa}>-<\imath \bar{c}_{\alpha} \imath c_{w}^{e} \imath \Phi_{\nu}><\imath \bar{c}_{\gamma} \imath c_{z}^{d} \imath \Phi_{\kappa}>\right] \\
& +<\imath \bar{c}_{v}^{f} \imath c_{w}^{e} \imath \Phi_{\alpha} \imath \Phi_{\nu}><\imath J_{\alpha} \imath J_{\kappa}><\imath \bar{c}_{\gamma} \imath c_{z}^{d} \imath \Phi_{\kappa}>-<\imath \bar{c}_{v}^{f} \imath c_{z}^{d} \imath \Phi_{\alpha} \imath \Phi_{\nu}><\imath J_{\alpha} \imath J_{\kappa}><\imath \bar{c}_{\gamma} \imath c_{w}^{e} \imath \Phi_{\kappa}> \\
& +<\imath \bar{c}_{v}^{f} \imath c_{\kappa} \imath \bar{c}_{\gamma} \imath c_{z}^{d}><\imath \bar{\eta}_{\kappa} \imath \eta_{\alpha}><\imath \bar{c}_{\alpha} \imath c_{w}^{e} \imath \Phi_{\nu}>-<\imath \bar{c}_{v}^{f} \imath c_{\kappa} \imath \bar{c}_{\gamma} \imath c_{w}^{e}><\imath \bar{\eta}_{\kappa} \imath \eta_{\alpha}><\imath \bar{c}_{\alpha} \imath c_{z}^{d} \imath \Phi_{\nu}> \\
& \left.+<\imath \bar{c}_{v}^{f} \imath c_{\mu} \imath \Phi_{\nu}><\imath \bar{\eta}_{\mu} \imath \eta_{\kappa}><\imath \bar{c}_{\kappa} \imath c_{w}^{e} \imath \bar{c}_{\gamma} \imath c_{z}^{d}>-<\imath \bar{c}_{v}^{f} \imath c_{w}^{e} \imath \bar{c}_{\gamma} \imath c_{z}^{d} \imath \Phi_{\nu}>\right\} \\
\tilde{\Gamma}_{\bar{c} ; \bar{c} c c \lambda}^{a d e g}(x, z, w, u)= & g f^{a b c} \frac{\delta^{3}}{\delta \imath \Phi_{\lambda u}^{g} \delta \imath c_{w}^{e} \delta \imath c_{z}^{d}}<\imath \bar{\eta}_{x}^{b} \imath \bar{\eta}_{x}^{c}>\left.\right|_{J=0} \\
= & g f^{a b c}<\imath \bar{\eta}_{x}^{b} \imath \eta_{\nu}><\imath \bar{\eta}_{x}^{c} \imath \eta_{\gamma}>\times \\
& \left\{2<\imath \bar{c}_{\nu} \imath c_{\mu} \imath \Phi_{\lambda u}^{g}><\imath \bar{\eta}_{\mu} \imath \eta_{\varepsilon}><\imath J_{\alpha} \imath J_{\kappa}>\left[<\imath \bar{c}_{\varepsilon} \imath c_{w}^{e} \imath \Phi_{\alpha}><\imath \bar{c}_{\gamma} \imath c_{z}^{d} \imath \Phi_{\kappa}>-<\imath \bar{c}_{\varepsilon} \imath c_{z}^{d} \imath \Phi_{\alpha}><\imath \bar{c}_{\gamma} \imath c_{w}^{e} \imath \Phi_{\kappa}>\right]\right. \\
& -2<\imath J_{\varepsilon} \imath \Phi_{\kappa}>\left[<\imath \bar{c}_{\nu} \imath c_{w}^{e} \imath \Phi_{\varepsilon} \imath \Phi_{\lambda u}^{g}><\imath \bar{c}_{\gamma} \imath c_{z}^{d} \imath \Phi_{\kappa}>-<\imath \bar{c}_{\nu} \imath c_{z}^{d} \imath \Phi_{\varepsilon} \imath \Phi_{\lambda u}^{g}><\imath \bar{c}_{\gamma} \imath c_{w}^{e} \imath \Phi_{\kappa}>\right] \\
& +2<\imath \bar{c}_{\nu} \imath c_{w}^{e} \imath \Phi_{\varepsilon}><\imath J_{\varepsilon} \imath J_{\mu}><\imath \Phi_{\mu} \imath \Phi_{\lambda u}^{g} \imath \Phi_{\alpha}><\imath J_{\alpha} \imath J_{\kappa}><\imath \bar{c}_{\gamma} \imath c_{z}^{d} \imath \Phi_{\kappa}> \\
& \left.-2<\imath \bar{c}_{\nu} \imath c_{\varepsilon} \imath \Phi_{\lambda u}^{g}><\imath \bar{\eta}_{\varepsilon} \imath \eta_{\kappa}><\imath \bar{c}_{\kappa} \imath c_{w}^{e} \imath \bar{c}_{\gamma} \imath c_{z}^{d}>+<\imath \bar{c}_{\nu} \imath c_{w}^{e} \imath \bar{c}_{\gamma} \imath c_{z}^{d} \imath \Phi_{\lambda u}^{g}>\right\}
\end{aligned}
$$

(In the above expression, Eq. (B.3), we omit the possible index (k) when the field type $\lambda$ refers to the $\vec{A}$-field for notational clarity.)

$$
\begin{aligned}
& 0=\int d^{4} x \delta\left(t-x_{0}\right)\left\{\left[\imath \partial_{x}^{0}<\imath \bar{c}_{r}^{h} \imath c_{u}^{g} \imath \bar{c}_{v}^{f} \imath c_{z}^{d} \imath \sigma_{x}^{e}>\right] \delta(w-x)-\left[\frac{\nabla_{i x}}{\left(-\nabla_{x}^{2}\right)}<\imath \bar{c}_{r}^{h} \imath c_{u}^{g} \imath \bar{c}_{v}^{f} \imath c_{z}^{d} \imath A_{i x}^{a}>\right]<\imath \bar{c}_{x}^{a} \imath c_{w}^{e}>\right. \\
& +\left[\imath \partial_{x}^{0}<\imath \bar{c}_{r}^{h} \imath c_{z}^{d} \imath \bar{c}_{v}^{f} \imath c_{w}^{e} \imath \sigma_{x}^{g}>\right] \delta(u-x)-\left[\frac{\nabla_{i x}}{\left(-\nabla_{x}^{2}\right)}<i \bar{c}_{r}^{h} \imath c_{z}^{d} \imath \bar{c}_{v}^{f} \imath c_{w}^{e} \imath A_{i x}^{a}>\right]<i \bar{c}_{x}^{a} \imath c_{u}^{g}> \\
& +\left[\imath \partial_{x}^{0}<\imath \bar{c}_{r}^{h} \imath c_{w}^{e} \imath \bar{c}_{v}^{f} \imath c_{u}^{g} \imath \sigma_{x}^{d}>\right] \delta(z-x)-\left[\frac{\nabla_{i x}}{\left(-\nabla_{x}^{2}\right)}<i \bar{c}_{r}^{h} \imath c_{w}^{e} \imath \bar{c}_{v}^{f} \imath c_{u}^{g} \imath A_{i x}^{a}>\right]<\imath \bar{c}_{x}^{a} \imath c_{z}^{d}> \\
& +<\imath \bar{c}_{v}^{f} \imath c_{z}^{d} \imath \sigma_{x}^{a}>\tilde{\Gamma}_{\sigma ; \bar{c} c c \bar{c}}^{a e g h}(x, w, u, r)-<\imath \bar{c}_{r}^{h} \imath c_{z}^{d} \imath \sigma_{x}^{a}>\tilde{\Gamma}_{\sigma ; \bar{c} c c \bar{c}}^{a e g f}(x, w, u, v) \\
& +<\imath \bar{c}_{v}^{f} \imath c_{w}^{e} \imath \sigma_{x}^{a}>\tilde{\Gamma}_{\sigma ; \bar{c} c c \bar{c}}^{a g d h}(x, u, z, r)-<\imath \bar{c}_{r}^{h} \imath c_{w}^{e} \imath \sigma_{x}^{a}>\tilde{\Gamma}_{\sigma ; \bar{c} c c \bar{c}}^{a g d f}(x, u, z, v) \\
& +<\imath \bar{c}_{v}^{f} \imath c_{u}^{g} \imath \sigma_{x}^{a}>\tilde{\Gamma}_{\sigma ; \bar{c} c c \bar{c}}^{a d \bar{c} h}(x, z, w, r)-<\imath \bar{c}_{r}^{h} \imath c_{u}^{g} \imath \sigma_{x}^{a}>\tilde{\Gamma}_{\sigma ; \bar{c} c c \bar{c}}^{a \operatorname{def}}(x, z, w, v) \\
& +<\imath \bar{c}_{v}^{f} \imath c_{z}^{d} \imath A_{i x}^{a}>\tilde{\Gamma}_{A ; \bar{c} c c \bar{c} i}^{a e g h}(x, w, u, r)-<i \bar{c}_{r}^{h} \imath c_{z}^{d} \imath A_{i x}^{a}>\tilde{\Gamma}_{A ; \bar{c} c c \bar{c} i}^{a e g f}(x, w, u, v) \\
& +<\imath \bar{c}_{v}^{f} \imath c_{w}^{e} \imath A_{i x}^{a}>\tilde{\Gamma}_{A ; \bar{c} c c \bar{c} i}^{a g d h}(x, u, z, r)-<i \bar{c}_{r}^{h} \imath c_{w}^{e} \imath A_{i x}^{a}>\tilde{\Gamma}_{A ; \bar{c} c c \bar{c} i}^{a g d f}(x, u, z, v) \\
& +<\imath \bar{c}_{v}^{f} \imath c_{u}^{g} \imath A_{i x}^{a}>\tilde{\Gamma}_{A ; \bar{c} c c \bar{c} i}^{a d e h}(x, z, w, r)-<\imath \bar{c}_{r}^{h} \imath c_{u}^{g} \imath A_{i x}^{a}>\tilde{\Gamma}_{A ; \bar{c} c c \bar{c} i}^{a d e f}(x, z, w, v) \\
& +\frac{1}{2}<\imath \bar{c}_{r}^{h} \imath c_{z}^{d} \imath \bar{c}_{v}^{f} \imath c_{x}^{a}>\left[\tilde{\Gamma}_{\bar{c} ; \bar{c} c c}^{a e g}(x, w, u)-2 g f^{a e g} \delta(w-x) \delta(u-x)\right] \\
& +\frac{1}{2}<\imath \bar{c}_{r}^{h} \imath c_{w}^{e} l \bar{c}_{v}^{f} \imath c_{x}^{a}>\left[\tilde{\Gamma}_{\bar{c} ; \bar{c} c c}^{a g d}(x, u, z)-2 g f^{a g d} \delta(u-x) \delta(z-x)\right] \\
& +\frac{1}{2}\left\langle\bar{c}_{r}^{h} \imath c_{u}^{g} \imath \bar{c}_{v}^{f} \imath c_{x}^{a}>\left[\tilde{\Gamma}_{\bar{c} ; \bar{c} c c}^{a d e}(x, z, w)-2 g f^{a d e} \delta(z-x) \delta(w-x)\right]\right. \\
& \left.+\frac{1}{2}<\bar{c}_{v}^{f} \imath c_{x}^{a}>\tilde{\Gamma}_{\bar{c} ; \bar{c} c c c \bar{c}}^{a \operatorname{degh}}(x, z, w, u, r)-\frac{1}{2}<\bar{c}_{r}^{h} \imath c_{x}^{a}>\tilde{\Gamma}_{\bar{c} ; \bar{c} c c c \bar{c}}^{a \operatorname{degf}}(x, z, w, u, v)\right\} . \\
& \tilde{\Gamma}_{\bar{c} ; \bar{c} c c c \bar{c}}^{a d e g f}(x, z, w, u, v)=g f^{a b c} \frac{\delta^{4}}{\delta \imath \bar{c}_{v}^{f} \delta \imath c_{u}^{g} \delta \imath c_{w}^{e} \delta \imath c_{z}^{d}}<\imath \bar{\eta}_{x}^{b} \bar{\eta}_{x}^{c}>\left.\right|_{J=0} \\
& =g f^{a b c}<\imath \bar{\eta}_{x}^{b} \imath \eta_{\mu}><\imath \bar{\eta}_{x}^{c} \imath \eta_{\nu}>\left\{-<\imath \bar{c}_{\mu} \imath c_{u}^{g} \bar{c}_{v}^{f} \imath c_{w}^{e} \imath \bar{c}_{\nu} \imath c_{z}^{d}>\right. \\
& +2<\imath \bar{\eta}_{\kappa} \imath \eta_{\varepsilon}>\left[<\imath \bar{c}_{v}^{f} \imath c_{\kappa} \imath \bar{c}_{\nu} \imath c_{z}^{d}><\imath \bar{c}_{\mu} \imath c_{u}^{g} \imath \bar{c}_{\varepsilon} \imath c_{w}^{e}>+ \text { c.p. }\left(c_{z}^{d}, c_{w}^{e}, c_{u}^{g}\right)\right] \\
& +2<\imath J_{\alpha} \imath J_{\beta}>\left[<\imath \bar{c}_{v}^{f} \imath c_{z}^{d} \imath \bar{c}_{\nu} \imath c_{u}^{g} \imath \Phi_{\beta}><\imath \bar{c}_{\mu} \imath c_{w}^{e} \imath \Phi_{\alpha}>+ \text { c.p. }\left(c_{z}^{d}, c_{w}^{e}, c_{u}^{g}\right)\right] \\
& +2<\imath J_{\alpha} \imath J_{\beta}><\imath J_{\gamma} \imath J_{\delta}>\left[<\imath \bar{c}_{v}^{f} \imath c_{z}^{d} \imath \Phi_{\gamma} \imath \Phi_{\beta}><\imath \bar{c}_{\mu} \imath c_{u}^{g} \imath \Phi_{\alpha}><\imath \bar{c}_{\nu} \imath c_{w}^{e} \imath \Phi_{\delta}>+ \text { c.p. }\left(c_{z}^{d}, c_{w}^{e}, c_{u}^{g}\right)\right] \\
& +2<\imath \bar{\eta}_{\kappa} \imath \eta_{\varepsilon}><\imath J_{\alpha} \imath J_{\beta}><\imath J_{\gamma} \imath J_{\delta}><\imath \bar{c}_{v}^{f} \imath c_{\kappa} \imath \Phi_{\beta}>\left[<\imath \bar{c}_{\mu} \imath c_{z}^{d} \imath \Phi_{\alpha}><\imath \bar{c}_{\nu} \imath c_{w}^{e} \imath \Phi_{\delta}><\imath \bar{c}_{\varepsilon} \imath c_{u}^{g} \imath \Phi_{\gamma}>+ \text { c.p. }\left(c_{z}^{d}, c_{w}^{e}, c_{u}^{g}\right)\right] \\
& +2<\imath \bar{\eta}_{\kappa} \imath \eta_{\varepsilon}><\imath J_{\alpha} \imath J_{\beta}><\imath J_{\gamma} \imath J_{\delta}><\imath \bar{c}_{v}^{f} \imath c_{\kappa} \imath \Phi_{\gamma}>\left[<\imath \bar{c}_{\mu} \imath c_{z}^{d} \imath \Phi_{\alpha}><\imath \bar{c}_{\nu} \imath c_{w}^{e} \imath \Phi_{\delta}><\imath \bar{c}_{\varepsilon} \imath c_{u}^{g} \imath \Phi_{\beta}>+ \text { c.p. }\left(c_{z}^{d}, c_{w}^{e}, c_{u}^{g}\right)\right] \\
& -2<\imath \bar{\eta}_{\kappa} \imath \eta_{\varepsilon}><\imath J_{\alpha} \imath J_{\beta}><\bar{c}_{v}^{f} \imath c_{\kappa} \imath \Phi_{\beta}>\left[<\imath \bar{c}_{\varepsilon} \imath c_{z}^{d} \imath \bar{c}_{\nu} \imath c_{u}^{g}><\imath \bar{c}_{\mu} \imath c_{w}^{e} \imath \Phi_{\alpha}>+ \text { c.p. }\left(c_{z}^{d}, c_{w}^{e}, c_{u}^{g}\right)\right]
\end{aligned}
$$




$$
\begin{aligned}
& +2<\imath \bar{\eta}_{\kappa} \imath \eta_{\varepsilon}><\imath J_{\alpha} \imath J_{\beta}>\times \\
& \left.\left[<\imath \bar{c}_{v}^{f} \imath c_{\kappa} \imath \bar{c}_{\nu} \imath c_{z}^{d}>\left(<\imath \bar{c}_{\mu} \imath c_{w}^{e} \imath \Phi_{\alpha}><\imath \bar{c}_{\varepsilon} \imath c_{u}^{g} \imath \Phi_{\beta}>-<\imath \bar{c}_{\mu} \imath c_{u}^{g} \imath \Phi_{\alpha}><\imath \bar{c}_{\varepsilon} \imath c_{w}^{e} \imath \Phi_{\beta}>\right)+\text { c.p. }\left(c_{z}^{d}, c_{w}^{e}, c_{u}^{g}\right)\right]\right\} .
\end{aligned}
$$

Note that in this expression, terms cyclic symmetric in the three ghost derivatives $\imath c_{z}^{d}, \imath c_{w}^{e}$ and $\imath c_{u}^{g}$ are denoted c.p. $\left(c_{z}^{d}, c_{w}^{e}, c_{u}^{g}\right)$.

[1] A. A. Slavnov, Theor. Math. Phys. 10, 99 (1972) [Teor. Mat. Fiz. 10, 153 (1972)].

[2] J. C. Taylor, Nucl. Phys. B 33, 436 (1971).

[3] J. S. Ball and T. W. Chiu, Phys. Rev. D 22, 2542 (1980).

[4] D. C. Curtis and M. R. Pennington, Phys. Rev. D 42, 4165 (1990).

[5] M. R. Pennington, arXiv:hep-th/9806200.

[6] R. Alkofer and L. von Smekal, Phys. Rept. 353, 281 (2001) [arXiv:hep-ph/0007355].

[7] W. Kummer, Acta Phys. Austriaca 41, 315 (1975).

[8] M. Baker, J. S. Ball and F. Zachariasen, Nucl. Phys. B 186, 531 (1981).

[9] G. B. West, Phys. Rev. D 27, 1878 (1983).

[10] S. Mandelstam, Phys. Rev. D 20, 3223 (1979).

[11] U. Bar-Gadda, Nucl. Phys. B 163, 312 (1980).

[12] L. von Smekal, A. Hauck and R. Alkofer, Annals Phys. 267, 1 (1998) [Erratum-ibid. 269, 182 (1998)] [arXiv:hep$\mathrm{ph} / 9707327]$.

[13] C. S. Fischer, J. Phys. G 32, R253 (2006) [arXiv:hep-ph/0605173].

[14] P. Watson, arXiv:hep-ph/9901454.

[15] P. Watson and R. Alkofer, Phys. Rev. Lett. 86, 5239 (2001) [arXiv:hep-ph/0102332].

[16] W. Schleifenbaum, A. Maas, J. Wambach and R. Alkofer, Phys. Rev. D 72, 014017 (2005) [arXiv:hep-ph/0411052].

[17] R. Oehme and W. Zimmermann, Phys. Rev. D 21, 471 (1980).

[18] T. Kugo and I. Ojima, Prog. Theor. Phys. Suppl. 66, 1 (1979); N. Nakanishi and I. Ojima, World Sci. Lect. Notes Phys. 27, 1 (1990).

[19] V. N. Gribov, Nucl. Phys. B 139 (1978) 1.

[20] D. Zwanziger, Nucl. Phys. B 485, 185 (1997) [arXiv:hep-th/9603203].

[21] D. Zwanziger, Nucl. Phys. B 518 (1998) 237.

[22] Ph. Boucaud, J. P. Leroy, A. Le Yaouanc, J. Micheli, O. Pene and J. Rodriguez-Quintero, JHEP 0806, 099 (2008) [arXiv:0803.2161 [hep-ph]].

[23] C. S. Fischer, A. Maas and J. M. Pawlowski, arXiv:0810.1987 [hep-ph].

[24] D. Epple, H. Reinhardt, W. Schleifenbaum and A. P. Szczepaniak, Phys. Rev. D 77, 085007 (2008) [arXiv:0712.3694 [hep-th]].

[25] C. Feuchter and H. Reinhardt, Phys. Rev. D 70 (2004) 105021 [arXiv:hep-th/0408236]; C. Feuchter and H. Reinhardt, arXiv:hep-th/0402106.

[26] E. S. Abers and B. W. Lee, Phys. Rept. 9, 1 (1973).

[27] P. Watson and H. Reinhardt, Phys. Rev. D 75, 045021 (2007) [arXiv:hep-th/0612114].

[28] P. Watson and H. Reinhardt, Phys. Rev. D 77, 025030 (2008) [arXiv:0709.3963 [hep-th]].

[29] P. Watson and H. Reinhardt, Phys. Rev. D 76, 125016 (2007) [arXiv:0709.0140 [hep-th]].

[30] C. Popovici, P. Watson and H. Reinhardt, arXiv:0810.4887 [hep-th].

[31] P. Watson and H. Reinhardt, arXiv:0711.2997 [hep-th].

[32] H. Reinhardt and P. Watson, arXiv:0808.2436 [hep-th].

[33] C. Itzykson and J. B. Zuber, "Quantum Field Theory", New York, USA: Mcgraw-Hill (1980) 705 P.(International Series in Pure and Applied Physics).

[34] W. J. Marciano and H. Pagels, Phys. Rept. 36, 137 (1978).

[35] S. K. Kim and M. Baker, Nucl. Phys. B 164, 152 (1980).

[36] J. S. Ball and T. W. Chiu, Phys. Rev. D 22, 2550 (1980) [Erratum-ibid. D 23, 3085 (1981)].

[37] H. Reinhardt and W. Schleifenbaum, arXiv:0809.1764 [hep-th]. 